\title{
Involvement of Cerebellar Neural Circuits in Active Avoidance Conditioning in Zebrafish
}

\author{
Wataru Koyama, ${ }^{1}$ Ryo Hosomi, ${ }^{1}$ Koji Matsuda, ${ }^{1}$ Koichi Kawakami, ${ }^{2}$ DMasahiko Hibi, ${ }^{1}$ and \\ Takashi Shimizu ${ }^{1}$
}

https://doi.org/10.1523/ENEURO.0507-20.2021

${ }^{1}$ Division of Biological Science, Graduate School of Science, Nagoya University, Nagoya, Aichi 464-8602, Japan and ${ }^{2}$ Laboratory of Molecular and Developmental Biology, National Institute of Genetics, and Department of Genetics, Graduate University for Advanced Studies (SOKENDAI), Mishima, Shizuoka 411-8540, Japan

\begin{abstract}
When animals repeatedly receive a combination of neutral conditional stimulus (CS) and aversive unconditional stimulus (US), they learn the relationship between CS and US, and show conditioned fear responses after CS. They show passive responses such as freezing or panic movements (classical or Pavlovian fear conditioning), or active behavioral responses to avoid aversive stimuli (active avoidance). Previous studies suggested the roles of the cerebellum in classical fear conditioning but it remains elusive whether the cerebellum is involved in active avoidance conditioning. In this study, we analyzed the roles of cerebellar neural circuits during active avoidance in adult zebrafish. When pairs of CS (light) and US (electric shock) were administered to wild-type zebrafish, about half of them displayed active avoidance. The expression of botulinum toxin, which inhibits the release of neurotransmitters, in cerebellar granule cells (GCs) or Purkinje cells (PCs) did not affect conditioning-independent swimming behaviors, but did inhibit active avoidance conditioning. Nitroreductase (NTR)mediated ablation of PCs in adult zebrafish also impaired active avoidance. Furthermore, the inhibited transmission of GCs or PCs resulted in reduced fear-conditioned Pavlovian fear responses. Our findings suggest that the zebrafish cerebellum plays an active role in active avoidance conditioning.
\end{abstract}

Key words: cerebellum; active avoidance; operant conditioning; botulinum toxin; nitroreductase; zebrafish

\section{Significance Statement}

An animal can associate a neutral conditioned stimulus and an aversive unconditioned stimulus, and escape to avoid an aversive stimulus. This is called active avoidance conditioning and is essential for an animal's survival. Although the amygdala and habenula nucleus are reportedly involved in active avoidance conditioning, the roles of other brain regions are largely unknown. We describe the roles of the cerebellum during active avoidance in adult zebrafish. The neurotoxin botulinum toxin-mediated inhibition of granule cells (GCs) or Purkinje cells (PCs), or the ablation of PCs, suppressed active avoidance conditioning. Our findings indicate that the cerebellum plays a positive role in active avoidance conditioning.

\section{Introduction}

An animal can associate two environmental stimuli and consequently shows certain behaviors. Broadly, there are neutral cues or conditional stimuli (CSs), such as sound or light, and aversive (noxious) cues or unconditional stimuli (USs), such as an electric shock. If an animal receives

\footnotetext{
Received November 24, 2020; accepted March 28, 2021; First published May $5,2021$.

The authors declare no competing financial interests.
}

repeated pairs of CSs and USs, it associates both to show fear responses on exposure to CSs. Consequently, CS-dependent aversive effects are expected. The animal may show two types of fear responses, "passive" and "active," on exposure to a CS. Passive responses are known as "classical fear conditioning" or "Pavlovian fear

Author contributions: T.S. and M.H. designed research; W.K., R.H., and K.M. performed research; K.K. contributed unpublished reagents/analytic tools; W.K. and R.H. analyzed data; M.H. wrote the paper. 
conditioning." This includes motor responses, including freezing behavior and panic movements (Sacchetti et al., 2002; Agetsuma et al., 2010; Amo et al., 2014; Lal et al., 2018), and autonomic reactions such as CS-evoked bradycardia responses (Supple and Leaton, 1990; Supple and Kapp, 1993; Supple et al., 1993; Yoshida and Hirano, 2010; Kotajima et al., 2014; Matsuda et al., 2017). It also shows active responses that result in the avoidance of noxious stimuli. Conditioning that elicits active (voluntary) responses is known as operant conditioning, and a type of operant conditioning that induces behaviors to avoid noxious stimuli is called "active avoidance" (Skinner, 1984). The amygdala in mammals is involved in both classical fear conditioning and active avoidance conditioning (Duvarci and Pare, 2014; Herry and Johansen, 2014). In zebrafish, a region in the dorsal telencephalon that is considered to be equivalent to the amygdala (Dm), and the habenula-raphe neural circuits, are reportedly involved in adaptive active avoidance conditioning (Aoki et al., 2013; Amo et al., 2014; Lal et al., 2018). However, it is still largely unknown if other neural circuits, including the cerebellum, are involved in fear conditioning.

The cerebellum plays important roles in some forms of motor coordination and motor learning, and is also involved in higher cognitive emotional functions (Ito, 2005, $2006,2008)$. The functions of the cerebellum rely on neural circuits that are conserved among vertebrates (Hashimoto and Hibi, 2012; Hibi et al., 2017). Purkinje cells (PCs) and granule cells (GCs) are major GABAergic and glutamatergic neurons in the cerebellum, and receive two inputs from outside of the cerebellum. PCs receive climbing fibers (CFs), which are axons from the inferior olivary nuclei (IOs). GCs receive mossy fibers (MFs) from precerebellar nuclei located in various regions of the brain. MF information is conveyed by GC axons, called parallel fibers (PFs). PCs integrate the two inputs and send outputs to the outside of the cerebellum through efferent neurons, which are deep cerebellar nuclei in mammals and eurydendroid cells in teleosts. The cerebellum of teleosts, including zebrafish, can be divided into the

This work was supported by the Ministry of Education, Culture, Sports, Science and Technology (MEXT) KAKENHI JP26115512 and the Japan Society for the Promotion of Science (JSPS) KAKENHI JP18H02448 (to M.H.), the JSPS KAKENHI JP18K06333 (to T.S.), the Core Research for Evolutional Science and Technology (CREST) Japan Science and Technology Agency (JST) JPMJCR1753 (to M.H.), and National Bioresource Project (NBRP) and NBRP/Fundamental Technologies Upgrading Program from Japan Agency for Medical Research and Development (AMED) (to K.K.).

K. Matsuda's present address: Center for Frontier Research, National Institute of Genetics, 1111 Yata, Mishima, Shizuoka 411-8540, Japan.

Acknowledgements: We thank the National Bioresource Project for providing transgenic fish, Kuniyo Kondoh and Yumiko Takayanagi for managing fish mating and care, and Hideyuki Tanabe for providing the method for active avoidance conditioning. We also thank the members of the Hibi Laboratory for helpful discussion.

Correspondence should be addressed to Takashi Shimizu at shimizu@ bio.nagoya-u.ac.jp or Masahiko Hibi at hibi@bio.nagoya-u.ac.jp.

https://doi.org/10.1523/ENEURO.0507-20.2021

Copyright $(\odot) 2021$ Koyama et al.

This is an open-access article distributed under the terms of the Creative Commons Attribution 4.0 International license, which permits unrestricted use, distribution and reproduction in any medium provided that the original work is properly attributed. rostro-medial domain [valvula cerebelli $(\mathrm{Va})$ and corpus cerebelli (CCe)] and the caudo-lateral domain [lobus caudalis cerebelli ( $\mathrm{LCa})$ and eminentia granularis (EG)] that are composed of different neural circuit structures (for review, see Hibi and Shimizu, 2012; Hibi et al., 2017). Cerebellar neural circuits are involved in classical conditioning, such as eye-blink conditioning. In mammals, lesions of the cerebellar vermis or IOs impair the acquisition of fear-conditioned bradycardia (Supple and Leaton, 1990; Kotajima et al., 2014). Inhibition of the vermis or efferent neurons with tetrodotoxin disrupts the consolidation of conditioned freezing responses in rats (Sacchetti et al., 2002). Lesions or chemical inhibition of the cerebellum also impair fear-conditioned bradycardia responses (Yoshida et al., 2004; Yoshida and Hirano, 2010). These studies suggest that the cerebellum is involved in classical fear conditioning. However, it is uncertain whether the cerebellar neural circuits control active avoidance conditioning.

Previous studies revealed that zebrafish can acquire classical fear conditioning and active avoidance conditioning from larval stages, although the timing to acquire robust conditioned behaviors varies depending on the experimental conditions (Aizenberg and Schuman, 2011; Valente et al., 2012; Matsuda et al., 2017; Lin et al., 2020). Zebrafish reliably show classical fear-conditioned bradycardia responses from the late larval stage $[\sim 20 \mathrm{~d}$ postfertilization (dpf); Matsuda et al., 2017]. Cerebellar neurons in the $\mathrm{CCe}$ are activated during conditioned behaviors and inhibition of the GC transmission prolonged recovery from the conditioned responses (Matsuda et al., 2017), implying a role of the cerebellar neural circuits in classical fear conditioning in zebrafish. In operant conditioning, cerebellar neurons are also activated, and lesions in the cerebellum delay decision-making (Lin et al., 2020). However, specific inhibition of cerebellar neurons in active avoidance conditioning has not been reported. In this study, we transgenically expressed botulinum toxin (BoTx), a neurotoxin that inhibits neurotransmitter release, in GCs or PCs, or nitroreductase (NTR), which can convert a prodrug metronidazole (MTZ) to a cytotoxin (Pisharath et al., 2007; Tabor et al., 2014), in PCs, to study the roles of cerebellar neural circuits in active avoidance conditioning in adult zebrafish.

\section{Materials and Methods}

\section{Ethics statement}

The animal experiments in this study were approved by the Institutional Animal Experiment Committee and were conducted in accordance with the Regulations on Animal Experiments at the institute.

\section{Zebrafish}

Wild-type zebrafish with the Oregon $A B$ genetic background and two previously reported transgenic $(\mathrm{Tg})$ lines, gSA2AzGFF152B (Takeuchi et al., 2015), which expresses a modified version of Gal4-VP16 (GAL4FF, also referred to as GFF) in the corpus cerebelli GCs, and $\operatorname{Tg}(\mathrm{UAS}$ : BoTxBLC-GFP) ${ }^{i c m 21}$, which expresses the light chain of 
BoTx in a GAL4-dependent manner (Sternberg et al., 2016), were used. To generate the Tg(aldoca:BoTxBLCGFP) line, the 5-kbp aldolase $\mathrm{Ca}$ (aldoca) promoter (Tanabe et al., 2010), the BoTxBLC-GFP gene (Sternberg et al., 2016), and the polyadenylation site (pAS) of pCS2+ were subcloned to the Tol2 vector pT2KDestRfaF (Nojima et al., 2010) by Gateway (Thermo Fisher Scientific). To generate the $\operatorname{Tg}(\mathrm{cb} / n 12: G a / 4 F F)$ line, the 2kbp cerebellin12 (cbln12) promoter (Dohaku et al., 2019), GAL4FF (Asakawa et al., 2008), and pAS of pCS2 + were subcloned to the Tol2 vector pT2ALR-Dest (Dohaku et al., 2019) by the Gateway system. To generate the Tg(aldoca:NTR-TagRFPT) line, the aldoca promoter, the epNTR-TagRFPT gene encoding a fusion protein of zebrafish codon-optimized enhanced-potency NTR (epNTR) and TagRFPT (Tabor et al., 2014), and pAS of pCS2+ were subcloned to pT2ARL-Dest by the Gateway system. To establish the Tg lines, $25 \mathrm{pg}$ of the Tol2 plasmid and $25 \mathrm{pg}$ of transposase-capped RNA were injected into one-cell-stage embryos. To inhibit GC transmission, gSA2AzGFF152B and Tg(cbln12:Gal4FF) were crossed with $T g(U A S: B O T x B L C-G F P)$. Tg adult fish that harbored both GAL4FF and GFP were identified before conditioning by genotyping with primer pairs: $5^{\prime}-$ AAGTGCGCCAAGTGTCTGAAGAAC-3' (F) and 5'-GAC CTGGACATGCTGCCTGCTGAT-3' (R) for GAL4FF, and 5'-CGAACATAGCTAGCGTGACCGTGA-3' (F) and 5'-TG GAGCACGTGTATCAGCTCATGC-3' (R) for BoTxBLC. Those that did not harbor GAL4FF or GFP were used as control fish. Similarly, adult $T g(a / d o c a: B o T x B L C-G F P)$ fish were identified by BoTxBLC genotyping. Adult fish that did not have the transgene were used as control fish. Fish were maintained in a 14/10 h light/dark cycle (light 9 A.M. to 11 P.M.; dark 11 P.M. to 9 A.M.) at $28.5^{\circ} \mathrm{C}$. Four- to 12 -month-old adult fish were used in this study. All experiments were conducted without distinction between males and females.

\section{MTZ treatment}

Adult Tg(aldoca:NTR-TagRFPT) fish (8-11 months old) were treated with $10 \mathrm{~mm}$ MTZ solution for $18 \mathrm{~h}$ and returned to their tank. Eleven days after treatment, the fish were subjected to behavior tests.

\section{Immunostaining and measurement}

Cryosections $14 \mu \mathrm{m}$ thick were prepared according to the previous publication (Bae et al., 2009). The sections were immunostained as previously described (Bae et al., 2009; Kani et al., 2010). The following antibodies were used: anti-GFP (1:1000, rat, Nacalai Tesque, catalog \#04404-84, RRID: $A B 10013361$ or 1:1000, rabbit, MBL International, catalog \#598, RRID: AB_591816) for BoTxBLC-GFP, antiNeurod1 (1:400, mouse, ascites; Kani et al., 2010), and antiparvalbumin 7 (1:1000, mouse monoclonal, ascites; Bae et al., 2009). The following secondary antibodies were used: Alexa Fluor 488 goat anti-rat $(\mathrm{H}+\mathrm{L}$, Invitrogen, Thermo Fisher Scientific, catalog \#A11006, RRID: AB_2534074), CF488A anti-rabbit $(\mathrm{H}+\mathrm{L}$, Biotium Inc., catalog \#20019, RRID: AB_10583180), and Alexa Fluor 568 goat anti- mouse IgG $(H+L$, Invitrogen, catalog \#A11031, RRID: $A B$ _144696). An LSM700 confocal laser-scanning microscope was used to obtain fluorescence images. GFP ${ }^{+}$ areas in the granular layer (GL) were measured by ImageJ software (https://imagej.nih.gov/ij/) (Table 1).

\section{Swimming performance test}

Swimming performance was analyzed as previously described (Matsuda et al., 2017). Freely swimming adult fish were recorded by a CMOS camera (30 frames per second; fps). The head position of each fish was tracked using the Tracker (http://physlets.org/tracker) program. The distance and direction of head movements between two consecutive frames were calculated in Microsoft Excel. An event showing $>90^{\circ}$ in the direction change of two consecutive movements was counted as one turn. Average swimming speed and turning frequencies were calculated in Microsoft Excel.

\section{Active avoidance conditioning, Pavlovian fear conditioning, and response to electric shocks}

Active avoidance conditioning was conducted by using a previously reported apparatus (Lal et al., 2018) and protocol (Aoki et al., 2013). Fish were maintained in a tank covered by white paper on the day before conditioning to prevent them from receiving special visual cues. For the conditioning, a white opaque tank ( $L 41 \mathrm{~cm} \times \mathrm{W} 17 \mathrm{~cm} \times$ $\mathrm{H} 12 \mathrm{~cm}$ ) with transparent walls at both ends and a trapezoidal wedge $(\mathrm{L} 10-20 \mathrm{~cm} \times \mathrm{W} 17 \mathrm{~cm} \times \mathrm{H} 5 \mathrm{~cm})$ in the center of the tank was used. For the Pavlovian fear conditioning, a side compartment of the tank for active avoidance conditioning was used $(\mathrm{L} 15.5 \mathrm{~cm} \times \mathrm{W} 17 \mathrm{~cm} \times$ $\mathrm{H} 12 \mathrm{~cm}$ ). Green LEDs (3.3-V DC, $2 \mathrm{~A})$ and a pair of platinum mesh electrodes (12-V AC: $0.71 \mathrm{~V} / \mathrm{cm}, 60 \mathrm{~Hz}$ ) were used to provide CS and US, respectively. Behaviors were monitored by a CMOS camera (30 fps). The timing of CS and US was controlled with a DAQ interface (USB-6008; National Instruments $\mathrm{Co}$ ) and laboratory-made software written in LabVIEW (National Instruments Co). Responses to electric shocks were examined by measuring swimming speed for $2 \mathrm{~s}$ before and after 10-s electric shocks after habituation for $20 \mathrm{~min}$.

\section{Statistics}

Data were analyzed and graphs were generated using GraphPad Prism (version 5.1) or the R software package (4.0.3; https://www.r-project.org/). Data are presented as the average \pm SEM. Statistical tests were applied as indicated in the figure legends. Additional statistical details are provided in Table 2.

\section{Results}

\section{Establishment of Tg zebrafish expressing botulinum toxin in GCs or PCs}

We previously used the $\mathrm{Tg}$ line gSA2AzGFF152B that expresses a modified version of Gal4-VP16 (Gal4FF) specifically in GCs to study the roles of GCs in classical fear conditioning (Takeuchi et al., 2015; Matsuda et al., 2017; 
Table 1: Tg lines and expression of BoTxBLC-GFP

\begin{tabular}{|c|c|c|c|c|c|}
\hline Tg lines & Expression & Sample number & Lateral sections (\%) & Medial sections (\%) & LCa (\%) \\
\hline gSA2AzGFF152B; & GCs (CCe $>>$ LCa, EG) & 1 & 14.7 & 69.5 & 0.2 \\
\hline Tg(UAS:BoTxBLC-GFP) & & 3 & 6.4 & 48.6 & 0.2 \\
\hline \multirow[t]{2}{*}{ Tg(UAS:BoTxBLC-GFP) } & & 2 & 37.7 & 42.1 & 55.0 \\
\hline & & 3 & 55.6 & 50.9 & 50.5 \\
\hline Tg(aldoca:BoTxBLC-GFP) & PCs & 1 & 96.4 & 97.7 & NA \\
\hline
\end{tabular}

Sagittal sections from three gSA2AzGFF152B;Tg(UAS:BoTxBLC-GFP) or Tg(cbln12:Gal4FF);Tg(UAS:BoTxBLC-GFP) adult fish were stained with anti-GFP and anti-Neurod1 antibodies. Sagittal sections from three $\mathrm{Tg}($ aldcoa:BoTxBLC-GFP) adult fish were stained with anti-GFP and anti-Pvalb7 antibodies. Two typical lateral and medial sections from each fish were used. For the GCs, the percentage of the GFP ${ }^{+}$area in the GL area was determined by using ImageJ software. For the PCs, the number of GFP ${ }^{+}$and Pvalb7 ${ }^{+}$cells was counted manually. The percentage of GFP ${ }^{+}$cells to Pvalb7 ${ }^{+}$cells was determined. CCe, corpus cerebelli; EG, eminentia granularis; GC, granule cells; LCa, lobus caudalis cerebelli; NA, not applicable; TL, torus longitudinalis.

Table 1). In addition to that line, we generated a Tg line, Tg(cbln12:Gal4FF), which expresses Gal4FF in GCs by using an $\sim 2.0$-kbp promoter/enhancer of the cerebellin12 (cbln12) gene, and reportedly drives transgene expression in GCs (Dohaku et al., 2019). We crossed gSA2AzGFF152B or Tg(cbln12:Gal4FF) with Tg(UAS: BoTxBLC-GFP), which expresses a fusion protein of the botulinum toxin light chain $B$ and green fluorescent protein (BoTxBLC-GFP), which inhibits the synaptic release of neurotransmitters, in a Gal4-dependent manner (Sternberg et al., 2016; Lal et al., 2018), and raised them to adulthood (hereafter referred as to 152B::BoTx and cbln12::BoTx lines; Fig. $1 A-G, H-N)$. We also generated a Tg line that expresses BoTxBLC-GFP in PCs by using an $\sim 5.0$-kbp promoter/enhancer of the aldolase Ca (aldoca) gene, which drives transgene expression specifically in PCs (Tanabe et al., 2010; Takeuchi et al., 2015; Dohaku et al., 2019; Tg(aldoca:BoTxBLC-GFP), hereafter referred as to aldoca:BoTx, Fig. 10-U). We found similar levels of BoTxBLC-GFP expression in the cerebellum of 5-dpf larvae of the same Tg lines. All of these $\mathrm{Tg}$ fish did not show any obvious abnormalities, including in swimming behaviors, during development. We dissected the brains from the adults of these Tg fish (more than three months old) after the behavior analyses described below and examined the expression of BoTxBLC-GFP by immunostaining with antibodies against GFP, and a GC marker (Neurod1; Kani et al., 2010) or a PC marker parvalbumin 7 (Pvalb7; Bae et al., 2009). Adult 152B::BoTx fish specifically showed BoTxBLC-GFP expression in GCs, mainly in the CCe (Fig. 1A-G), as reported previously for late-stage larvae (Matsuda et al., 2017). Adult cbln12::BoTx fish displayed BoTxBLC-GFP expression in GCs in both the $\mathrm{CCe}$ and LCa, as well as in GCs in the torus longitudinalis of the mesencephalon (TL; Fig. $1 H-N$ ). cbln12::BoTx fish also displayed BoTxBLC-GFP expression in some telencephalic neurons (data not shown) as the $c b / n 12$ promoter was reported to drive transgene expression in telencephalic neurons in addition to GCs (Dohaku et al., 2019). In 152B::BoTx and cbln12::BoTx fish, BoTxBLCGFP mRNA was transcribed in the somata of GCs while BoTxBLC-GFP protein was transported to the GC axons. Consistent with this, BoTxBLC-GFP was also detected in the molecular layer (ML) of the cerebellum and/or the stratum marginale (SM) of the optic tectum where GC axons were present (Fig. 1G,I,N). A comparison with Neurod1 expression revealed that $60.7 \%$ and $11.6 \%$ of $\mathrm{GCs}$ in the medial and lateral domains, respectively, of the CCe expressed BoTxBLC-GFP in 152B::BoTx fish while only $0.433 \%$ of GCs in the $\mathrm{LCa}$ expressed BoTxBLC-GFP. In cbln12::BoTx fish, 43.8\% and $42.9 \%$ of $\mathrm{GCs}$ in the medial and lateral regions, respectively, of the CCe, and $49.9 \%$ of GCs in the $\mathrm{LCa}$, expressed BoTxBLC-GFP (Table 1). These data suggest that the 152B::BoTx fish preferentially expressed BoTxBLC-GFP in GCs of the medial CCe whereas cbln12B::BoTx expressed it in GCs of both the CCe and LCa. A comparison with Pvalb7 expression indicated that $97.7 \%$ of Pvalb7 ${ }^{+}$PCs expressed BoTxBLC-GFP in the aldoca:BoTx line (Table 1). Although we cannot exclude the possibility that BoTx was also expressed in some neurons other than PCs, they are likely to be a minor population. Our data indicate that BoTx was expressed in large numbers of GCs or PCs of the cerebellum of these $\mathrm{Tg}$ fish.

\section{Active avoidance conditioning in adult zebrafish}

We employed an assay system for two-way active avoidance learning by using a tank with two compartments (Lal et al., 2018) and a previously published protocol (Aoki et al., 2013). In this system, the two compartments were separated by a fixed trapezoidal wedge allowing fish to move freely from one compartment to the other (Fig. 2A). Light exposure to green LEDs and electric shocks were considered as the CS and US, respectively. After habituation for $20 \mathrm{~min}$, when a fish was located in a compartment, it was exposed to CS for $15 \mathrm{~s}$ during the training session. When the fish did not escape to the other side after $10 \mathrm{~s}$, a 5-s US was applied (Fig. 2B). A 15- to 20-s interval was inserted between trials. When the fish escaped before US during the presentation of CS, it was considered as a successful trial (Movie 1). When the fish did not escape before US, it was considered as a failed trial (Movie 2). We found that no fish $(n=43)$ escaped to the other side within the first $10 \mathrm{~s}$ after the onset of CS during the first trial before 
Table 2: Summary of statistical analyses

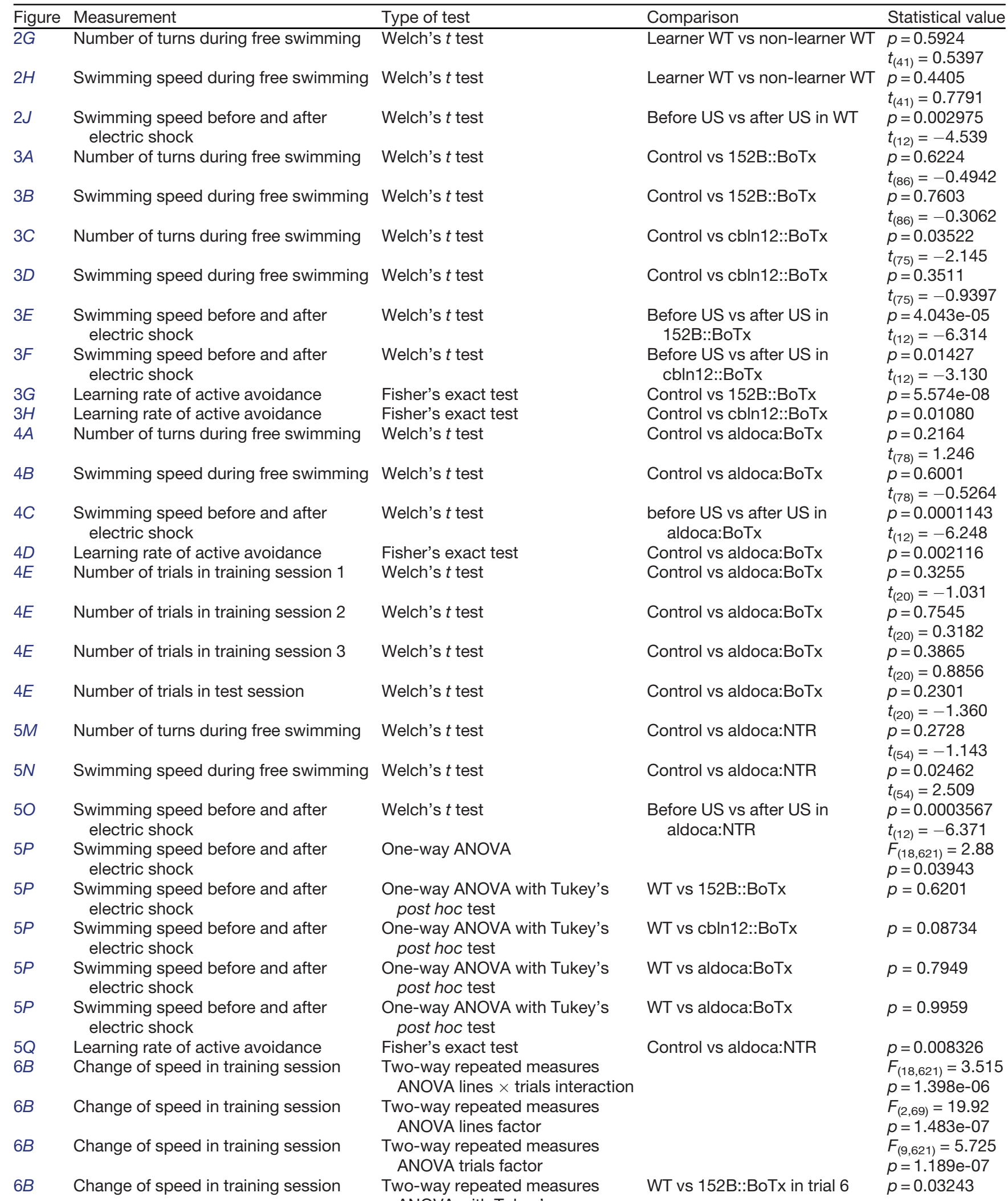


Table 2: Continued

\begin{tabular}{|c|c|c|c|c|}
\hline Figure & Measurement & Type of test & Comparison & Statistical value \\
\hline & & $\begin{array}{l}\text { Two-way repeated measures } \\
\text { ANOVA with Tukey's } \\
\text { post hoc test }\end{array}$ & & \\
\hline $6 B$ & Change of speed in training session & $\begin{array}{l}\text { Two-way repeated measures } \\
\text { ANOVA with Tukey's } \\
\text { post hoc test }\end{array}$ & WT vs 152B::BoT in trial 8 & $p=4.1 \mathrm{e}-09$ \\
\hline $6 B$ & Change of speed in training session & $\begin{array}{l}\text { Two-way repeated measures } \\
\text { ANOVA with Tukey's } \\
\text { post hoc test }\end{array}$ & WT vs aldoca:BoTx in trial 8 & $p=2.6 \mathrm{e}-05$ \\
\hline $6 B$ & Change of speed in training session & $\begin{array}{l}\text { Two-way repeated measures } \\
\text { ANOVA with Tukey's } \\
\text { post hoc test }\end{array}$ & WT vs 152B::BoTx in trial 9 & $p=0.005936$ \\
\hline $6 B$ & Change of speed in test session & $\begin{array}{l}\text { Two-way repeated measures } \\
\text { ANOVA lines } \times \text { trials } \\
\text { interaction }\end{array}$ & & $\begin{array}{l}F_{(18,621)}=1.083 \\
p=0.3649\end{array}$ \\
\hline $6 B$ & Change of speed in test session & $\begin{array}{l}\text { Two-way repeated measures } \\
\text { ANOVA lines factor }\end{array}$ & & $\begin{array}{l}F_{(2,69)}=28.35 \\
p=1.398 \mathrm{e}-06\end{array}$ \\
\hline $6 B$ & Change of speed in test session & $\begin{array}{l}\text { Two-way repeated measures } \\
\text { ANOVA trials factor }\end{array}$ & & $\begin{array}{l}F_{(9,621)}=1.431 \\
p=0.171\end{array}$ \\
\hline $6 B$ & Change of speed in training session & $\begin{array}{l}\text { Two-way repeated measures } \\
\text { ANOVA with Tukey's } \\
\text { post hoc test }\end{array}$ & WT vs 152B::BoTx & $p<1 e-22$ \\
\hline $6 B$ & Change of speed in training session & $\begin{array}{l}\text { One-way ANOVA with Tukey's } \\
\text { post hoc test }\end{array}$ & WT vs aldoca:BoTx & $p<1 e-22$ \\
\hline $6 B$ & Change of speed in training session & $\begin{array}{l}\text { One-way ANOVA with Tukey's } \\
\text { post hoc test }\end{array}$ & $\begin{array}{l}\text { aldoca:BoTx vs } \\
\text { 152B::BoTx }\end{array}$ & $p=0.9425$ \\
\hline $6 C$ & $\begin{array}{l}\text { Learning rate of Pavlovian fear } \\
\text { conditioning }\end{array}$ & $\begin{array}{l}\text { Fisher's exact test with BH } \\
\text { post hoc test }\end{array}$ & WT vs 152B::BoTx & $p=0.02892$ \\
\hline $6 C$ & $\begin{array}{l}\text { Learning rate of Pavlovian fear } \\
\text { onditioning }\end{array}$ & $\begin{array}{l}\text { Fisher's exact test with } \mathrm{BH} \\
\text { post hoc test }\end{array}$ & WT vs aldoca:BoTx & $p=0.02892$ \\
\hline $6 C$ & $\begin{array}{l}\text { Learning rate of Pavlovian fear } \\
\text { conditioning }\end{array}$ & $\begin{array}{l}\text { Fisher's exact test with } \\
\text { BH post hoc test }\end{array}$ & $\begin{array}{l}\text { 152B::BoTx vs } \\
\text { aldoca:BoTx }\end{array}$ & $p=1.000$ \\
\hline
\end{tabular}

In all figures, the data distribution was normal. WT, wild-type.

receiving any US. Fish that had eight successful trials among 10 consecutive trials were considered to have established active avoidance in the session, that session was terminated, and the fish were subjected to the next session following a 20 min interval (Fig. 2C). Each session contained up to 60 trials. The fish that did not establish active avoidance within 60 trials were not subjected to further trials, while those that established active avoidance in three consecutive sessions (sessions 1-3) were subjected to the test session in which only CSs were provided (Fig. 2C). When the fish succeeded in eight trials among the 10 consecutive trials within 60 trials, they were considered as learners. Fish that did not establish active avoidance in either training or test sessions were considered as non-learners. A small number of fish that established active avoidance in the training sessions failed in the test session $(n=$ 3/43). It is currently unknown why these fish failed after the establishment of active avoidance. In this experimental condition, $51.2 \%(n=43)$ of wild-type adult fish succeeded in establishing active avoidance learning (Fig. 2D). Learner fish established active avoidance in $32.5 \pm 3.2,20.4 \pm 2.2,13.6 \pm 1.1$, and $10.5 \pm 0.5$ trials (average $\pm \mathrm{SE}$ ) in training sessions 1, 2, 3, and the test session, respectively (Fig. 2E). Although we could not determine exactly when learners responded to CS to escape, we found that learner fish escaped from the compartment where they received CS at $4.0 \pm 0.63$, $4.6 \pm 0.44,5.0 \pm 0.60$, and $4.0 \pm 0.59 \mathrm{~s}$ (average $\pm \mathrm{SE}$ ) after receiving $\mathrm{CS}$ in training sessions 1, 2, 3, and the test session, respectively (Fig. $2 F$ ). We examined average swimming speed and turn frequency during $1 \mathrm{~min}$ of free swimming after the habituation session and before the training session. They were not significantly different between learners and non-learners (Fig. 2G,H). The data indicate that about half of the adult zebrafish could acquire active avoidance conditioning and progressively improved active avoidance. Our findings further suggest that the ability of zebrafish to acquire active avoidance conditioning was not directly related to their ability to swim. Furthermore, non-learner fish did not show a freezing response, i.e., reduction of swimming speed after CS (Fig. 2/), indicating that the freezing response was not the cause of the failure to learn.

\section{Inhibition of GC transmission suppresses active avoidance conditioning}

We next analyzed the GC-silenced 152B::BoTx and cbln12::BoTx fish, and compared them with control siblings that did not have Gal4FF and/or BoTxBLC-GFP 


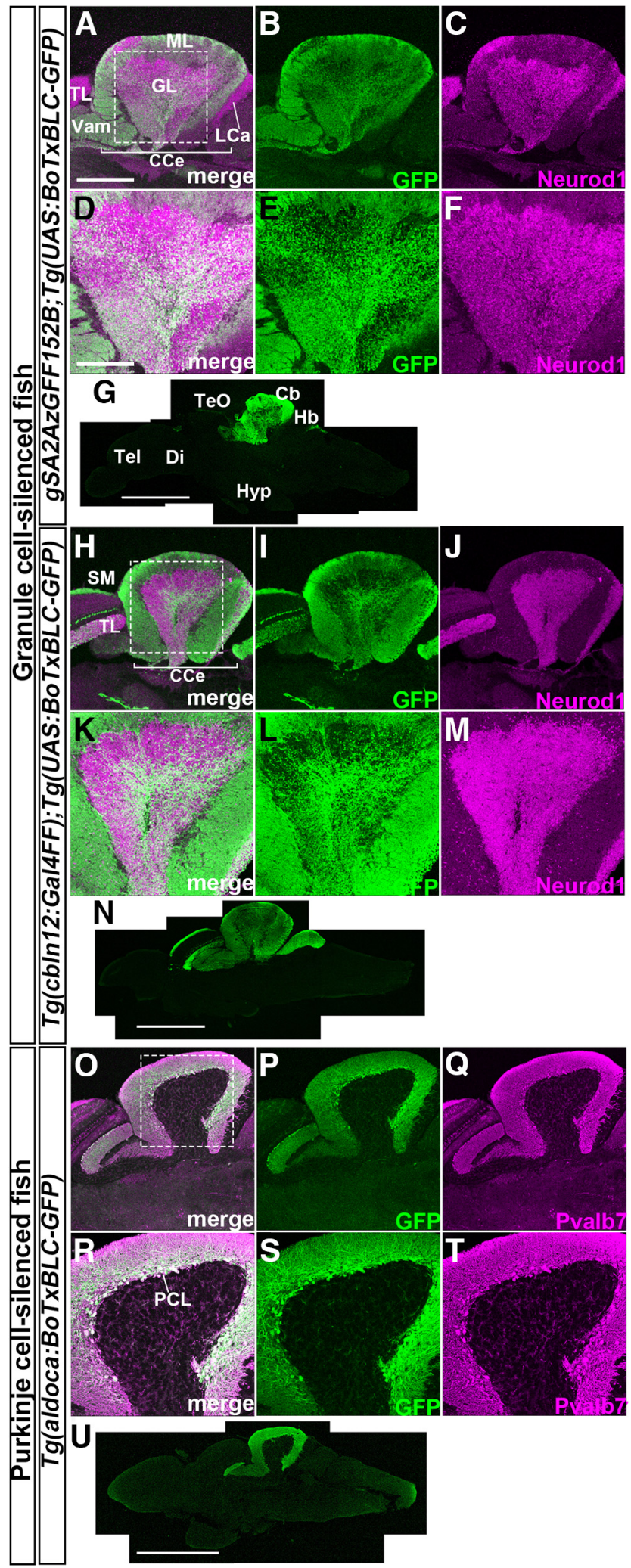

Figure 1. Establishment of Tg fish that express botulinum toxin in GCs or PCs. Sagittal sections of adult gSA2AzGFF152B;Tg(UAS: BoTxBLC-GFP) (A-G), Tg(cbln12:Gal4FF);Tg(UAS:BoTxBLC-GFP) $(\boldsymbol{H}-\mathbf{N})$, and Tg(aldoca:BoTxBLC-GFP) (O-U) brains were stained with anti-GFP (green), and anti-Neurod1, or anti-parvalbumin 7 (Pvalb7, magenta) antibodies. $\boldsymbol{A}-\boldsymbol{C}, \boldsymbol{H}-\boldsymbol{J}, \boldsymbol{O}-\boldsymbol{Q}$, Cerebellum region. genes (Fig. 3). Although the cerebellum is involved in some forms of motor control, there was no significant difference in the average swimming speed and turn frequency between 152B::BoTx and control fish (Fig. $3 A, B$ ). Although turn frequency was slightly lower in cbln12::BoTx fish than in control fish (Fig. 3C), average swimming speed was not significantly different between cbln12::BoTx and control fish (Fig. 3D). Both 152B::BoTx and cbln12::BoTx fish responded to electric shocks as did wild-type fish (Figs. 2J, 3E,F), suggesting that the expression of BoTx did not strongly affect their swimming behaviors or response to US. When 152B::BoTx and control fish were subjected to active avoidance conditioning, $44.2 \%(n=43)$ of control fish were learners while no $(n=45) 152 B$ ::BoTx fish were learners (Fig. 3G). When cbln12::BoTx and control fish were examined, $55.3 \%(n=38)$ of control fish were learners while $25.6 \%(n=39)$ of cbln12::BoTx fish were learners (Fig. $3 H$ ). The data indicate that the inhibition of neurotransmitter release in the GCs suppressed active avoidance conditioning. A relatively lower suppression of learner ratio observed in the cbln12::BoTx fish may be because of a relatively lower expression of BoTxBLC-GFP at the single-cell level in cbln12::BoTx fish than in 152B::BoTx fish (Table 1). The data indicate that the BoTx-mediated inhibition of GC transmission suppressed active avoidance conditioning without strongly inhibiting the swimming behaviors or aversive stimuli-dependent escape responses.

\section{Inhibition of PC transmission suppresses active avoidance conditioning}

We then analyzed the PC-silenced aldoca:BoTx fish, and compared them with control siblings that did not have the transgene. The average swimming speed and turn frequency were not significantly different between aldoca:BoTx and control fish (Fig. 4A,B). The aldoca:BoTx fish responded to electric shocks in a manner smilar to wild-type fish (Figs. 2J, 4C). When fish were subjected to active avoidance conditioning, $45.7 \%$ of control sibling fish $(n=35)$ were learners whereas $13.3 \%(n=45)$ of aldoca: BoTx fish were learners (Fig. 4D). Furthermore, although there was no statistically significant difference, the aldoca:BoTx learner fish took more trials than control sibling fish to establish active avoidance in training session 1 and in the test session (Fig. 4E). These data indicate that inhibition of neurotransmitter release in PCs suppressed active avoidance conditioning but did not significantly affect conditioning-independent swimming behaviors or aversive stimuli-dependent escape responses.

continued

$\boldsymbol{D}-\boldsymbol{F}, \boldsymbol{K}-\boldsymbol{M}, \boldsymbol{R}-\boldsymbol{T}$, High-magnification views of the boxes in $\boldsymbol{A}, \boldsymbol{H}, \boldsymbol{O}$. $\boldsymbol{G}, \boldsymbol{N}, \boldsymbol{U}$, Low-magnification views. $\mathrm{Cb}$, cerebellum; CCe, corpus cerebelli; Di, diencephalon; GL, granular layer; Hb, hindbrain; Hyp, hypothalamus; LCa, lobus caudalis cerebelli; ML, molecular layer; PCL; PC layer; SM, stratum marginale; Tel, telencephalon; TeO, tectum opticum; TL, torus longitudinalis; Vam, medial division of valvula cerebelli. Scale bars: $400 \mu \mathrm{m}(\boldsymbol{A}$; applies to $\boldsymbol{A}-\boldsymbol{C}, \boldsymbol{H}-\boldsymbol{J}, \mathbf{O}$ $\boldsymbol{Q}), 200 \mu \mathrm{m}$ (D; applies to $\boldsymbol{D}-\boldsymbol{F}, \boldsymbol{K}-\boldsymbol{M}, \boldsymbol{R}-\boldsymbol{T}), 1 \mathrm{~mm}(\boldsymbol{G}, \boldsymbol{N}, \boldsymbol{U})$. 
A
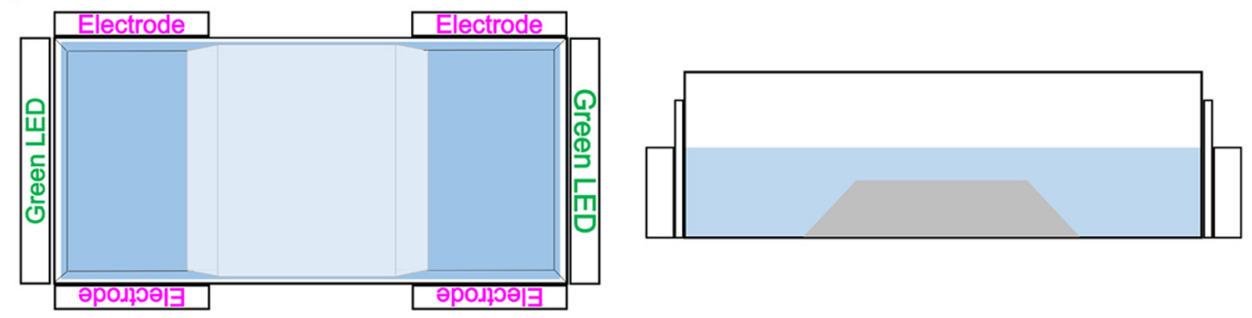

B
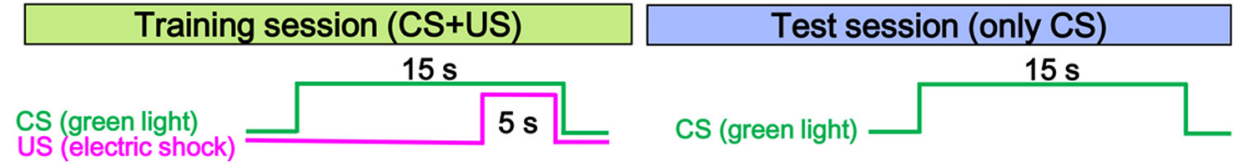

C

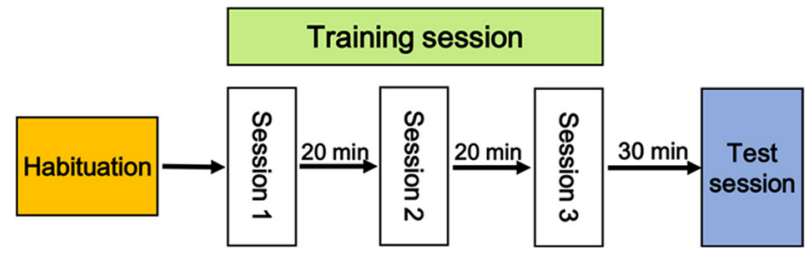

D

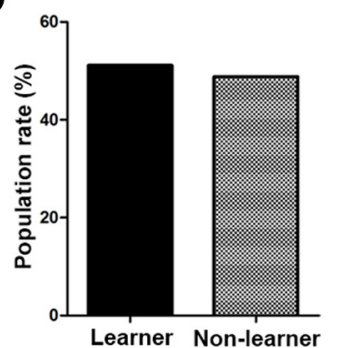

E

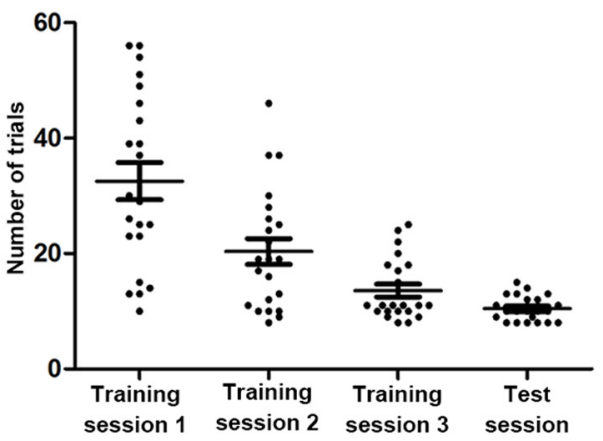

G

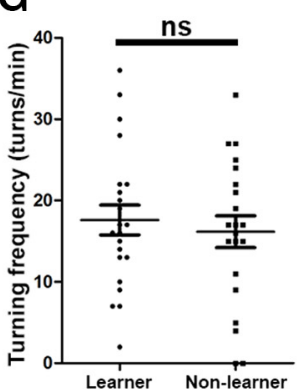

H

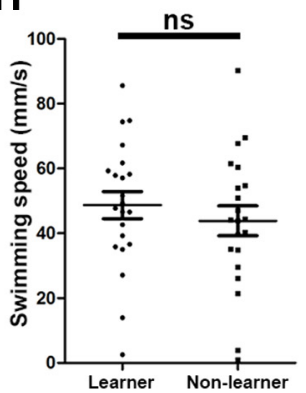

F
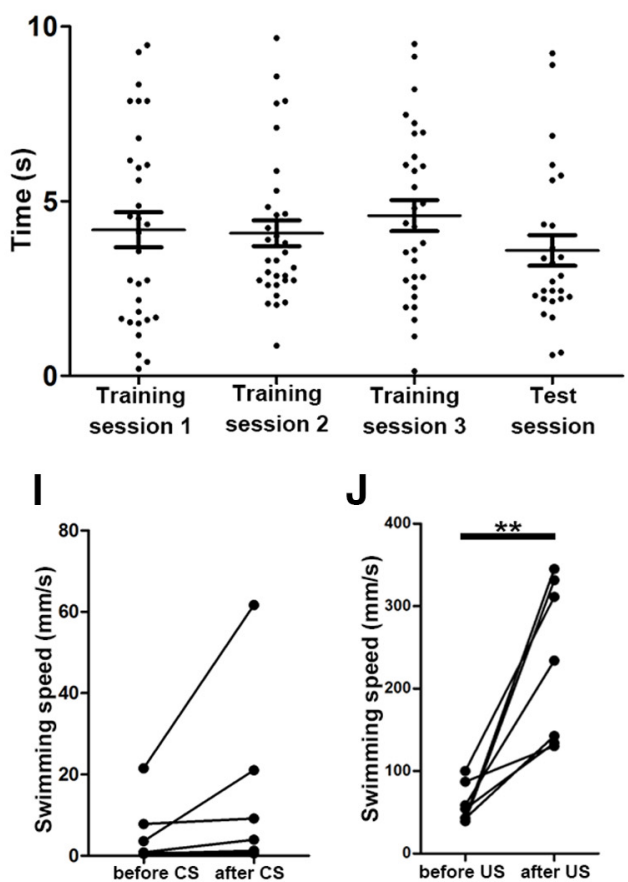

J

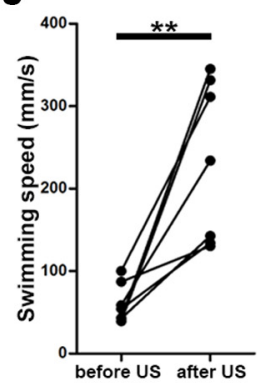

Figure 2. Active avoidance conditioning of wild-type fish. $\boldsymbol{A}$, Tank used for active avoidance conditioning. A white opaque tank (L41 $\mathrm{cm} \times \mathrm{W} 17 \mathrm{~cm} \times \mathrm{H} 12 \mathrm{~cm}$ ) with transparent walls at both ends, and a trapezoidal wedge (L10-20 cm $\times \mathrm{W} 17 \mathrm{~cm} \times \mathrm{H} 5 \mathrm{~cm})$ in the center, were used. Green LEDs and a pair of electrodes were placed on each side. Top view (left panel) and side view (right panel). $\boldsymbol{B}$, $\boldsymbol{C}$, Protocol for active avoidance. In the habituation session, a fish was allowed to swim freely for 20 min in the tank. In the training session, when a fish was located in a side compartment, the LED was turned on for $15 \mathrm{~s}$ (CS). If the fish did not escape to the other side after $10 \mathrm{~s}$, an electric shock was administered for $5 \mathrm{~s}$ (US) in each trial. When the fish moved before the electric shocks, the trial was successful and was followed by a 30-s interval and the next trial. When fish had eight successful trials among 10 consecutive trials, they were considered to have established active avoidance in the training session, and were subjected to the next trial session. When fish did not establish active avoidance within 60 trials, the training session was terminated. When fish established active avoidance in three consecutive training sessions, they were subjected to the test session. In the test session, only light stimuli 
continued

with LEDs were administered. When the fish had eight successful trials among 10 consecutive trials in the training session, they were considered to be learners. When fish did not establish active avoidance in the training session or did not succeed in the test session, they were considered to be non-learners. $\boldsymbol{D}$. Acquisition of active avoidance conditioning in wild-type adult fish. Percentages of learners and non-learners are indicated $(n=43)$. $\boldsymbol{E}$, Number of trials when learner fish established active avoidance in the training and test sessions $(n=22)$. The graph shows averages and SEs of the data. $\boldsymbol{F}$, Time from CS to escape in each session of learner fish $(n=7)$. The graph shows averages and SEs of the data. $\boldsymbol{G}, \boldsymbol{H}$, Swimming behaviors. Turning frequency (turns/min) and swimming speed $(\mathrm{mm} / \mathrm{s})$ of learners and non-learners during free swimming (learner; $n=22$, non-learner; $n=21)$. The graph shows averages and SEs of the data (ns indicates non-significance, Welch's $t$ test). I, Freezing response of non-learners. Average swimming speed $(\mathrm{mm} / \mathrm{s})$ of seven non-learners before and after the onset of CS in the 44th-53rd trials of training session 1 was calculated. $\boldsymbol{J}$, Test for responsiveness to electric shocks in wild-type adult fish $(n=7)$. Swimming speed for $2 \mathrm{~s}$ before and after electric shocks was calculated $\left({ }^{* *} p<0.01\right.$, Welch's $t$ test). ns, not significant.

\section{Ablation of PCs in adult fish suppresses active avoidance conditioning}

Since BoTx was expressed in 152B::BoTx, cbln12:: BoTx and aldoca:BoTx fish from an early larval stage, rewiring of cerebellar neural circuits might occur and compensate for the deficiency of GC/PC transmission during development. We ablated PCs in adult fish with NTR. We established a $\mathrm{Tg}$ line that expresses a fusion protein of modified NTR and TagRFP-T (NTR-TagRFPT; Tabor et al., 2014) in PCs by using the aldoca promoter/enhancer [Tg (aldoca:NTR-TagRFPT)]. NTR-TagRFPT was expressed from early larval stages to adult stages (Fig. $5 A, B, D, E$ ). NTR-TagRFPT signals completely overlapped with Pvalb7 signals in the cerebellum but not in the optic tectum where Pvalb7-expressing Type I neurons are present (Fig. 5C), indicating that NTR-TagRFPT was specifically expressed in PCs. We found that $11 \mathrm{~d}$ after treatment with MTZ, most NTR-TagRFPT-positive and Pvalb7-positive cells disappeared from the cerebellum although Pvalb7-positive Type I neurons remained in the optic tectum while the ML was abrogated (Fig. 5G-L), indicating that most PCs were ablated. Turn frequency and swimming speed were reduced in PC-ablated fish compared with control wild-type fish (the difference in turn frequency was not statistically significant; Fig. $5 \mathrm{M}$, $N)$. However, the PC-ablated fish responded to electric shocks, as did wild-type, 152B::BoTx, cbln12::BoTx,

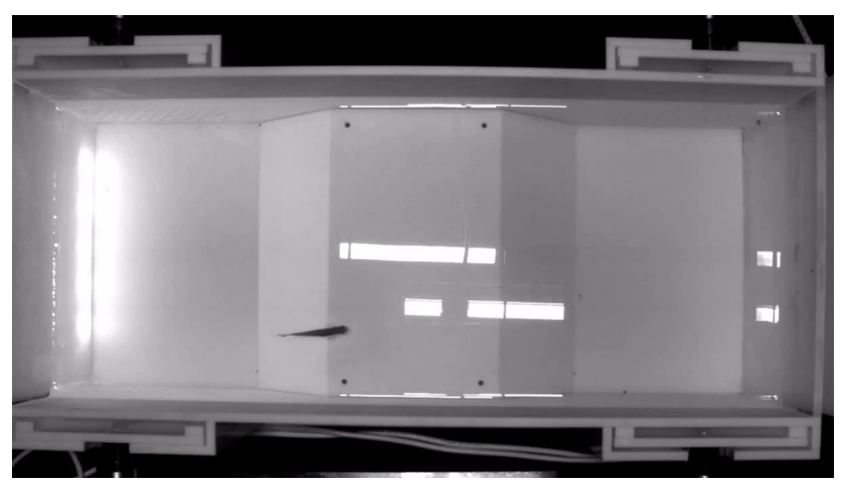

Movie 1. Active avoidance of a wild-type fish, successful trial. A successful trial of active avoidance of a wild-type learner fish in the 25th trial of training session 1 is shown. The timing of the CS (light exposure with a green LED) and US (electric shock) are indicated. Note that the fish responded to the CS and escaped into the compartment on the other side of the tank. [View online] and aldoca:BoTx fish, and swam similarly to wild-type fish after electric shocks (Figs. 2J, 5O,P), suggesting that the acute ablation of PCs slightly affected free swimming but not aversive stimuli-dependent swimming. In active avoidance conditioning, $51.2 \%$ of control fish $(n=43)$ became learners whereas only $7.69 \%$ of PC-ablated fish $(n=13)$ were learners (Fig. $5 Q)$, indicating that the ablation of PCs in the adult cerebellum also perturbed active avoidance conditioning.

\section{Inhibition of GC/PC transmission also suppresses Pavlovian fear conditioning}

Although the inhibition of GCs or PCs suppressed active avoidance conditioning, it is not clear whether it inhibited classical fear conditioning. We conducted Pavlovian fear conditioning in which we determined CS-evoked panic movements by using 152B::BoTx (GC-silenced) and aldoca:BoTx (PC-silenced) fish. In the Pavlovian fear conditioning, light exposure to green LEDs was provided for $2 s$ as a CS in the habituation session (10 trials), electric shocks were delivered as 0.2 -s USs with CSs after the onset of 1.8-s CS in the training session (10 trials), and only CSs were administered in the test session (10 trials; Fig. 6A). We assessed the Pavlovian fear conditioned responses by measuring changes in swimming speed for a total of $3 \mathrm{~s}$, i.e., $1.5 \mathrm{~s}$ before and after the onset of CS. Wild-type fish progressively increased CSevoked changes in swimming speed in the training

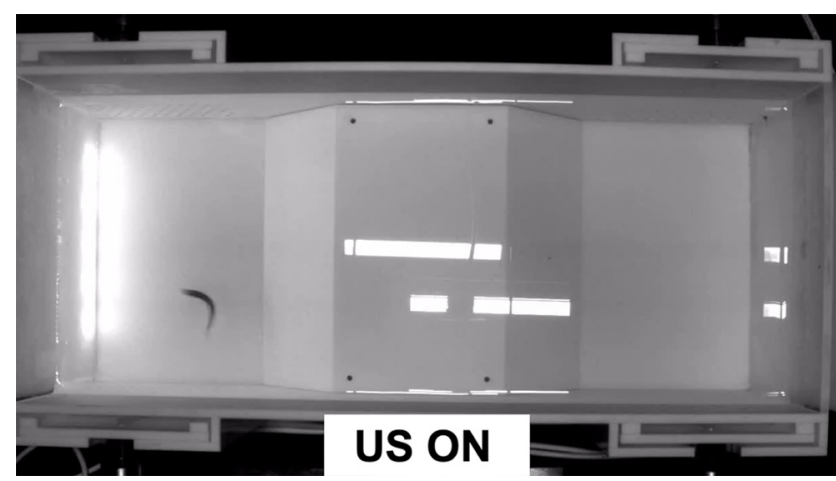

Movie 2. Active avoidance of a wild-type fish, failed trial. A failed trial of active avoidance of a wild-type non-learner fish in the 25th trial of training session 1 is shown. The timing of the CS and US are indicated. Note also that the fish did not escape after the presentation of CS but responded to US. [View online] 

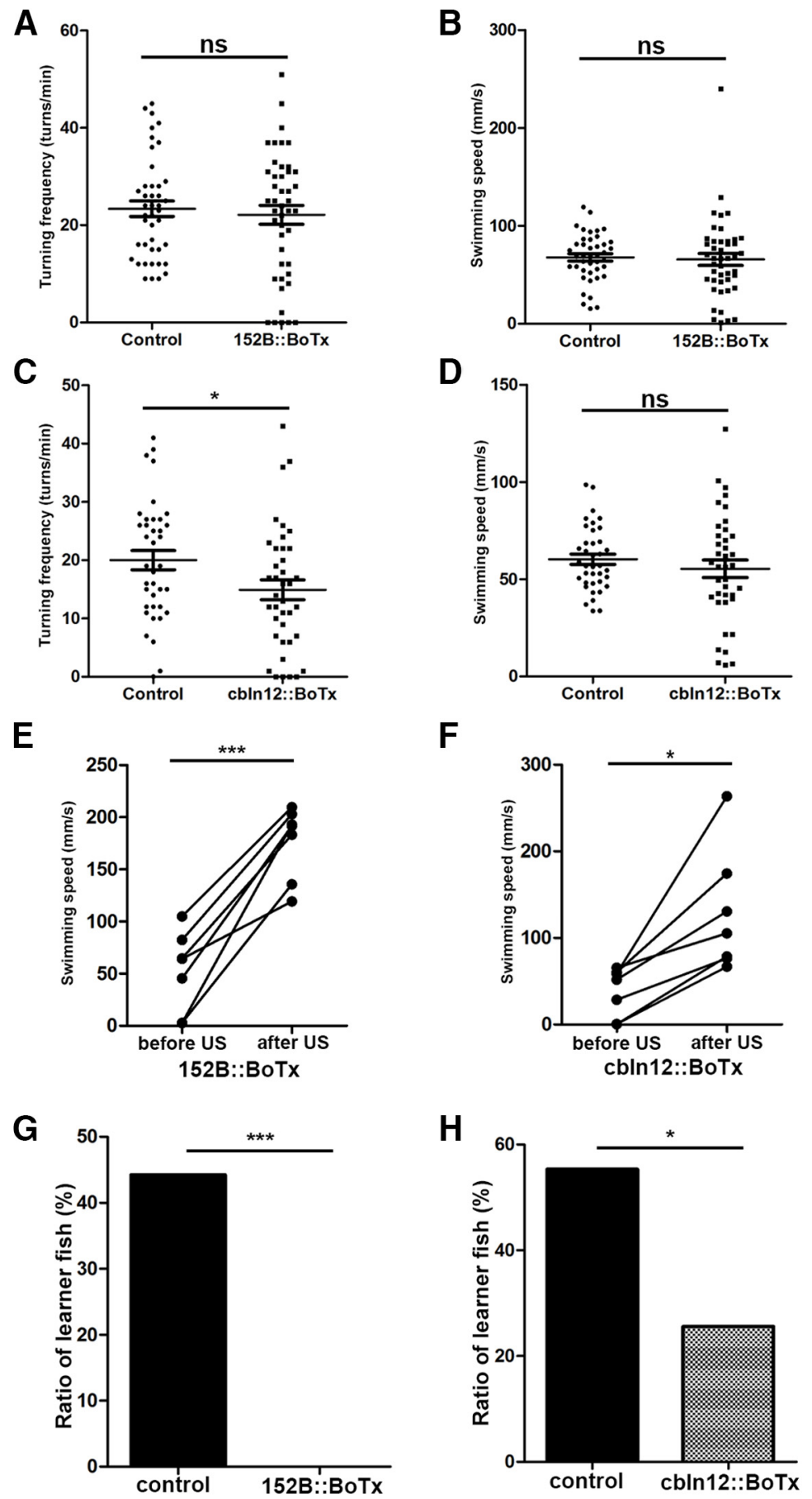

Figure 3. Expression of botulinum toxin in GCs suppresses active avoidance conditioning. $\boldsymbol{A}, \boldsymbol{B}$, Turning frequency (turns/min) and swimming speed (mm/s) of gSA2AzGFF152B;Tg(UAS:BoTxBLC-GFP) (152B::BoTx) and control sibling fish during free swimming (152B::BoTx; $n=47$, control; $n=43$ ). The graph shows the averages and SEs of the data (ns indicates non-significance, Welch's $t$ test. $\boldsymbol{C}$, $\boldsymbol{D}$, Turning frequency and swimming speed of Tg(cbln12:Gal4FF); Tg(UAS:BoTxBLC-GFP) (cbln12::BoTx) fish during free swimming (cbln12::BoTx; $n=39$, control; $n=38$ ). The graph shows the averages and SEs of the data (ns indicates non-significance, ${ }^{*} p<0.05$, Welch's $t$ test). $\boldsymbol{E}, \boldsymbol{F}$, Response to electric shocks in 152B::BoTx $(n=7)$ and cbln12::BoTx $(n=7)$ fish. Swimming speed was calculated for $2 \mathrm{~s}$ before and after the electric shocks $\left({ }^{\star \star *} p<0.001,{ }^{*} p<0.05\right.$, Welch's $t$ test). $\mathbf{G}, \boldsymbol{H}$, Percentages of active avoidance learners for 152B::BoTx $(n=47)$ and control sibling fish $(n=43 ; \boldsymbol{G})$, and for cbln12::BoTx $(n=39)$, and control sibling fish $\left(n=38 ; \boldsymbol{H} ;{ }^{\star \star *} p<0.001,{ }^{\star} p<0.05\right.$, Fisher's exact test). ns, not significant.

session, and moved quickly after CS in the test session (Fig. 6B; Movie 3). The CS-evoked responses were gradually reduced in the test session (Fig. 6B). In contrast, the conditioned responses of 152B::BoTx and aldoca:
BoTx fish barely improved in the training session (Fig. $6 B$ ). The conditioned responses in the test session were significantly weaker in 152B::BoTx and aldoca:BoTx fish than in wild-type fish (Fig. 6B; Movies 4, 5). We defined 
A
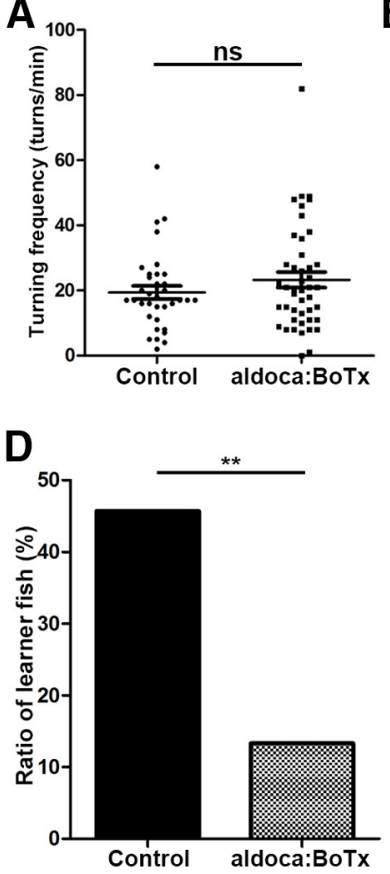

B

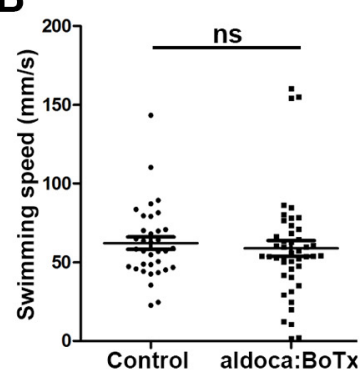

E

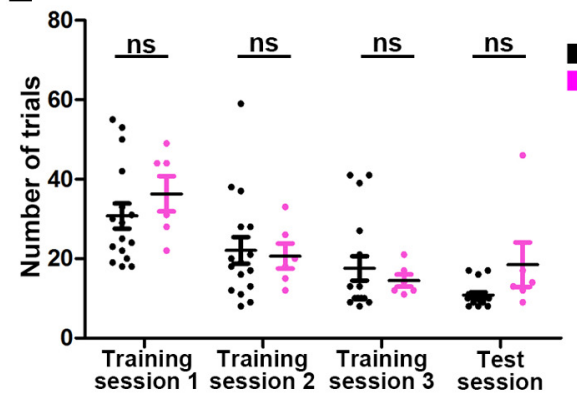

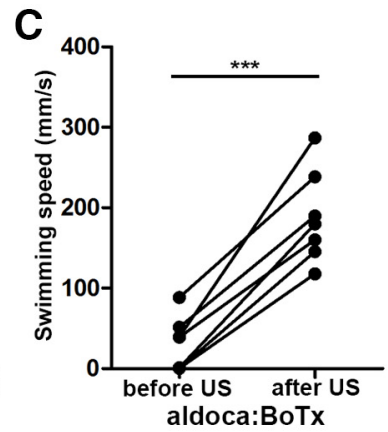

Control aldoca:BoTx

Figure 4. Expression of botulinum toxin in PCs suppresses active avoidance conditioning. $\boldsymbol{A}, \boldsymbol{B}$, Turning frequency (turns/min) and swimming speed (mm/s) of Tg(aldoca:BoTxBCL-GFP) (aldoca:BoTx) and control sibling fish during free swimming (aldoca:BoTx; $n=45$, control; $n=35$ ). The graph shows the averages and SEs of the data (ns indicates non-significance, Welch's $t$ test). $\boldsymbol{C}$, Response to electric shocks in aldoca:BoTx fish $(n=7)$. Swimming speed was calculated for $2 \mathrm{~s}$ before and after the electric shocks $\left({ }^{* \star *} p<0.001\right.$, Welch's $t$ test). $\boldsymbol{D}$, Percentages of active avoidance learners of aldoca:BoTx $(n=45)$ and control $(n=35)$ fish $\left({ }^{\star *} p<0.01\right.$, Fisher's exact test). $\boldsymbol{E}$, Number of trials required to establish active avoidance conditioning (aldoca:BoTx: $n=6$; control: $n=16)$. The graph shows the averages and SEs of the data (ns indicates non-significance, Welch's $t$ test).

"learners" as fish whose conditioned responses were significantly higher in the test session than in the habituation session; $57.1 \%(n=14)$ of wild-type fish were learners whereas a significantly lower number of 152B::BoTx (8.33\%, $n=12)$ and aldoca:BoTx $(7.69 \%, n=13)$ fish were leaners (Fig. 6C). These data indicate that the inhibition of GC or PC transmission also perturbed the Pavlovian fear conditioning. We further examined Pavlovian fear responses by measuring changes in speed for $1.5 \mathrm{~s}$ before and after the onset of CS in active avoidance conditioning; $70 \%(n=10)$ of wild-type fish showed CS-evoked quick movements (Fig. 6D), indicating that Pavlovian fear conditioning was also established during active avoidance conditioning.

\section{Discussion}

\section{Genetic inhibition of cerebellar neurons in zebrafish}

The roles of cerebellar neural circuits in fear conditioning have traditionally been studied by inhibiting the cerebellum with physical or laser-induced lesions, or by local administration of an anesthetic drug (Yoshida et al., 2004; Yoshida and Hirano, 2010; Ahrens et al., 2012; Lin et al., 2020). In this study, we inhibited neurotransmitter release from GCs or PCs by expressing BoTx using the promoter/enhancer of GC-expressed or PC-expressed genes (i.e., cbln12 and aldoca) or a Gal4 trap line that drives transgene expression in GCs (Fig. 1). These genetic inhibitions are thought to be reliable, reproducible, and cell-type-specific as the neurotoxin was stably expressed in the cells of interest. The Gal4-UAS system induces transgene expression more strongly than when driven directly by celltype-specific enhancer/promoters. However, methylation-mediated silencing of UAS-regulated transgenes occurs more as generations progress, resulting in variegated transgene expression (Akitake et al., 2011). Although the gSA2AzGFF152B line expresses Gal4FF in most GCs of the medial CCe (Takeuchi et al., 2015) and the cbln12 promoter/enhancer drives transgene expression in all differentiated GCs (Dohaku et al., 2019), the Gal4-dependent expression of BoTxBLCGFP was mosaic (Fig. 1; Table 1). In contrast, the expression of BoTxBLC-GFP, which was driven directly by the aldoca promoter/enhancer (Tanabe et al., 2010), was detected in most, if not all, PCs (Fig. 1; Table 1). Therefore, careful examination of BoTx expression is required to validate results from neuronal inhibition with Gal4-UAS-mediated toxin expression. In this study, even when BoTx was expressed in about half of the GCs of the medial CCe in 152B::BoTx and cbln12::BoTx fish, this suppressed active avoidance conditioning (Fig. 3), suggesting that the establishment of active avoidance conditioning is sensitive to the number of functional GCs in the cerebellum. cbln12:: BoTx expressed BoTxBLC-GFP in neurons in the telencephalon and GCs in the TL, in addition to GCs in the cerebellum (Fig. 1). Although the roles of these neurons outside the cerebellum are not necessarily excluded, it is likely that 

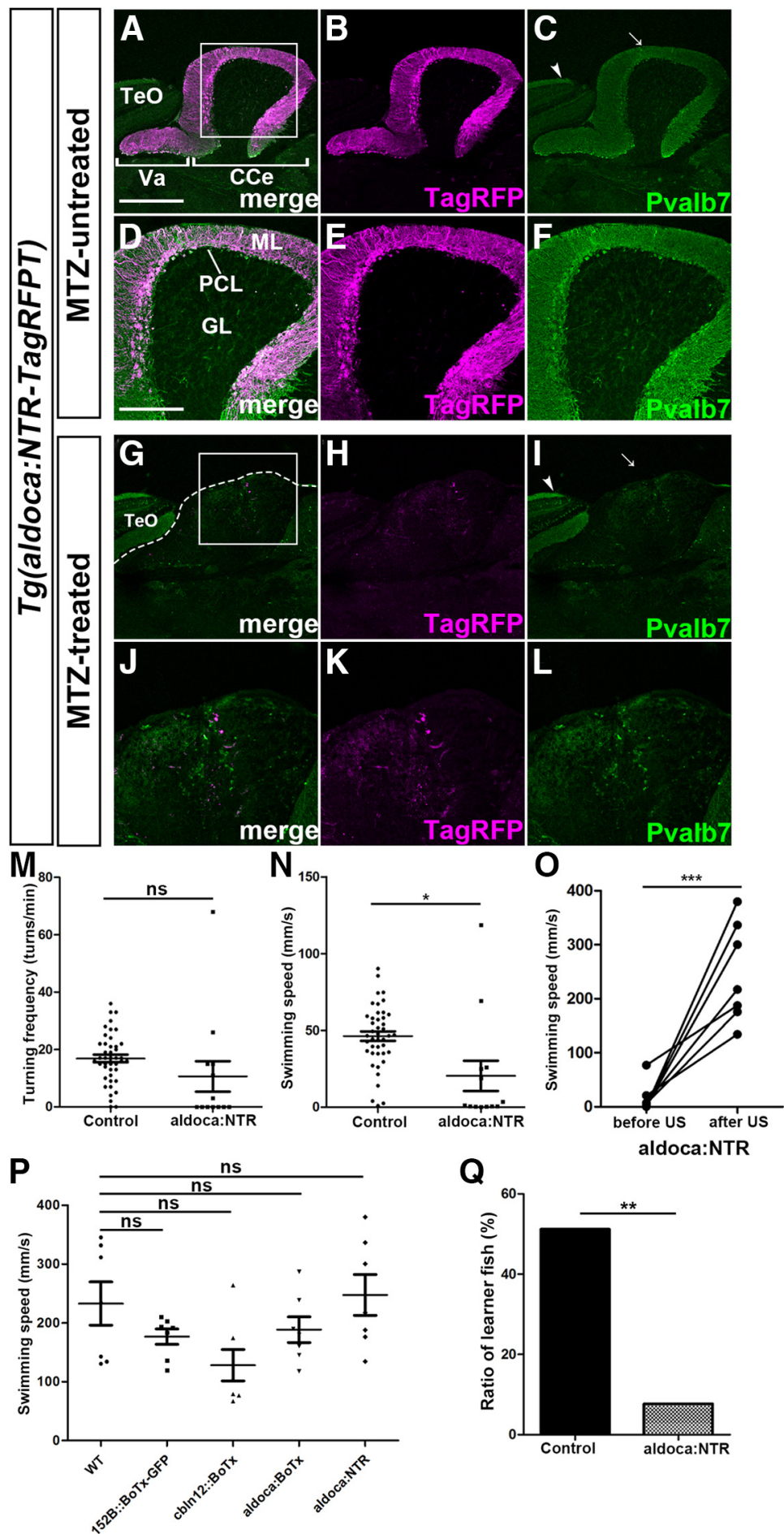

Q

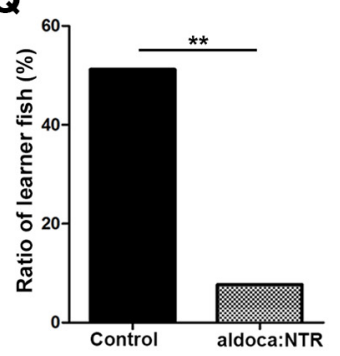

Figure 5. NTR-mediated ablation of PCs in adult fish suppresses active avoidance conditioning. $\boldsymbol{A}-\boldsymbol{L}$, Ablation of PCs. Adult Tg(aldoca:NTR-TagRFPT) fish were treated with MTZ for $18 \mathrm{~h}(\boldsymbol{A}-\boldsymbol{F})$ or left untreated $(\mathbf{G}-\mathbf{L})$. The fish were subjected to behavior assays and subsequent histologic analysis $11 \mathrm{~d}$ after MTZ treatment. Sagittal sections were stained with anti-Pvalb7 antibody (green). Expression of NTR-TagRFPT (TagRFP, magenta) is also shown. $\boldsymbol{D}-\boldsymbol{F}, \boldsymbol{J}-\boldsymbol{L}$, High-magnification views of the boxes in $\boldsymbol{A}$, $\boldsymbol{G}$. Arrows and arrow heads indicate Pvalb7-positive dendrites of PCs (in the cerebellum) and Type I neurons (in the optic tectum), respectively. The dotted line in $\mathbf{G}$ indicates the limit of the cerebellum. Note that the Pvalb7 signal in PCs but not in Type I neurons disappeared

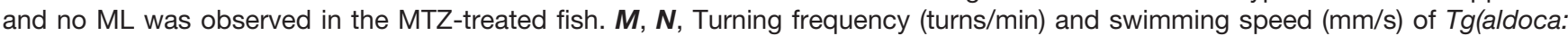
NTR-TagRFPT) (aldoca:NTR) and control fish during free swimming (aldoca:NTR; $n=13$, control; $n=43$ ). The graph shows the averages and SEs of the data (ns indicates non-significance, ${ }^{*} p<0.05$, Welch's $t$ test). $\boldsymbol{O}$, Response to electric shocks in adult aldoca: NTR fish treated with MTZ $(n=7)$. Swimming speed was calculated for $2 \mathrm{~s}$ before and after the electric shocks $\left({ }^{\star \star *} p<0.001\right.$, Welch's $t$ test). $\boldsymbol{P}$, Swimming speed for $2 \mathrm{~s}$ after US in each strain. The graph shows the averages and SEs of the data (ns indicates 
continued

non-significance, one-way repeated measures ANOVA with Tukey's post hoc test). $\boldsymbol{Q}$, Percentage of active avoidance learners of aldoca:NTR $(n=13)$ and control wild-type $(n=43)$ fish ${ }^{* \star} p<0.01$, Fisher's exact test). Va, valvula cerebelli. The other abbreviations are described in Figure 1. Scale bars: $400 \mu \mathrm{m}(\boldsymbol{A}$; applies to $\boldsymbol{A}-\boldsymbol{C}, \boldsymbol{G}-\boldsymbol{I})$ and $200 \mu \mathrm{m}(\boldsymbol{D}$; applies to $\boldsymbol{D}-\boldsymbol{F}$, $\boldsymbol{J}-\boldsymbol{L})$. ns, not significant.

A

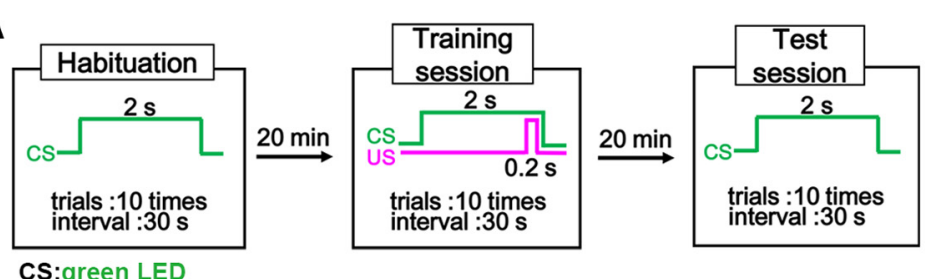

US:electric shock

B

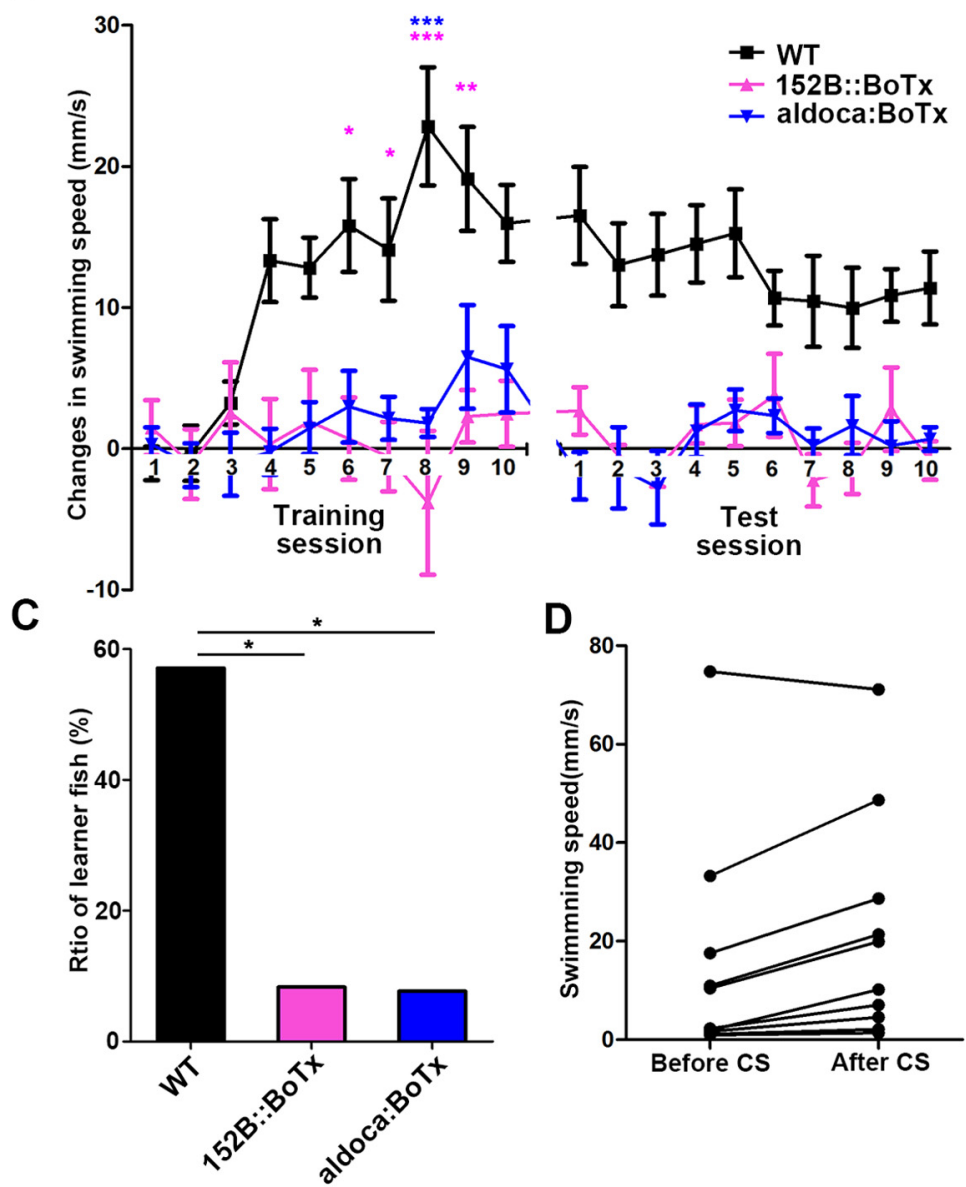

Figure 6. Expression of botulinum toxin in GCs or PCs also perturbs classical conditioning responses. $\boldsymbol{A}$, Protocol for classical fear conditioning. A compartment on one side of the tank in Figure $2 A$ was used. In the habituation session, a light stimulus (CS) was provided for $2 \mathrm{~s}$ per trial (10 trials). In the training session, a paired CS and US (0.2-s electric shock given $1.8 \mathrm{~s}$ after the onset of CS) was administered in each trial (10 trials). In the test session, CS alone was administered (10 trials). The interval between trials was $30 \mathrm{~s}$, and the interval between sessions was $20 \mathrm{~min}$. B, Changes in swimming speed before and after the CS of wild-type (WT, $n=25), 152 \mathrm{~B}::$ BoTx $(n=23)$, and aldoca:BoTx $(n=24)$ fish. Swimming speed was measured for $1.5 \mathrm{~s}$ before and after the CS in each trial, and average changes in swimming speed in the training and test sessions were calculated. The graph shows the averages and SEs of the data (Training session; lines factor: $p=1.483 \mathrm{e}-07$, trials factor: $p=1.189 \mathrm{e}-07$, lines $\times$ trials interaction: $p=1.398 \mathrm{e}-06$, two-way repeated measures ANOVA; ${ }^{\star \star \star} p<0.001$, ${ }^{\star *} p<0.01,{ }^{*} p<0.05$, two-way repeated measures ANOVA with Tukey's post hoc test. Test session; lines factor: $p=1.398 \mathrm{e}-06$, trials factor: $p=0.171$, lines $\times$ trials interaction: $p=0.3649$, two-way repeated measures ANOVA; WT vs 152B::BoTx in test session: $p<1 \mathrm{e}-22$, WT vs aldoca:BoTx in test session: $p<1$ e-22, 152B::BoTx vs aldoca:BoTx in test session: $p=0.9425$, one-way ANOVA with Tukey's post hoc test). $\boldsymbol{C}$, Percentages of Pavlovian conditioning learners of WT $(n=14), 152 \mathrm{~B}:$ BoTx $(n=12)$, and aldoca:BoTx $(n=13)$ fish $\left({ }^{*} p<0.05\right.$, Fisher's exact test with BH post hoc test). $D$, Pavlovian panic responses during active avoidance conditioning. Data from 10 wild-type fish that were subjected to active avoidance conditioning were used. Since wild-type fish established active avoidance in the 13th trial at the earliest, swimming speed for $1.5 \mathrm{~s}$ before and after the CS was measured in each trial from the fourth to the 13th trial. Average speed is plotted in the graph. 


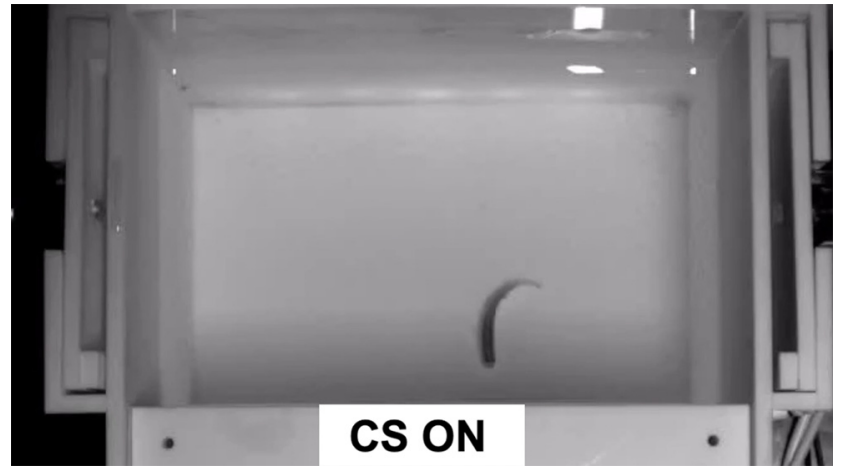

Movie 3. Pavlovian fear conditioning of a wild-type fish. Behavior of a wild-type learner fish in trial 1 of the test session is shown. The timing of the CS is indicated. Note that the fish moved quickly after the presentation of CS. [View online]

the inhibition of GCs in the cerebellum had a major impact on active avoidance conditioning in both 152B::BoTx and cbln12::BoTx fish. The BoTx-mediated inhibition of PCs also perturbed active avoidance conditioning (Fig. 4). Collectively, BoTx expression driven by both Gal4-UAS or a cell-specific promoter/enhancer successfully inhibited the transmission of GCs and PCs.

PC-silenced fish did not show abnormal locomotion but generated an erratic form of body displacement in some conditions (Chang et al., 2020). However, both GC-silenced and PC-silenced fish did not show conditioning-independent swimming and responded to US in a manner similar to wild-type fish (Figs. 3, 4). Thus, although the BoTx-mediated inhibition of GCs or PCs might affect smooth and/or well-coordinated movements to some extent, its main impact on conditioning is likely to be independent of abnormal swimming behavior.

In this study, BoTx was expressed from early larval stages when GCs and PCs had differentiated. Therefore, physical or functional rewiring of neural circuits might occur and compensate for the deficiency of GC or PC transmission. It might alleviate the effects of inhibition of GCs or PCs. To address this issue, we specifically ablated PCs in the adult cerebellum using the NTR-MTZ system

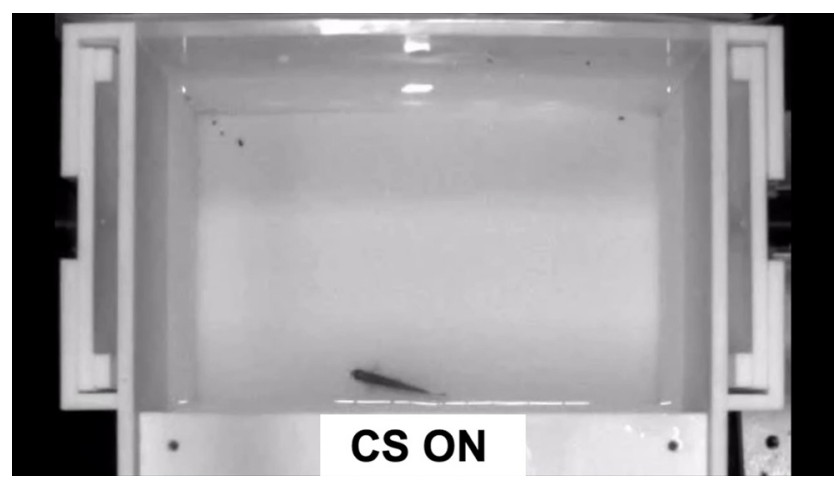

Movie 4. Pavlovian fear conditioning of a 152B::BoTx fish. Behavior of a 152B::BoTx non-learner fish in trial 1 of the test session is shown. The timing of the CS is indicated. Note that the fish did not move after the presentation of CS. [View online]

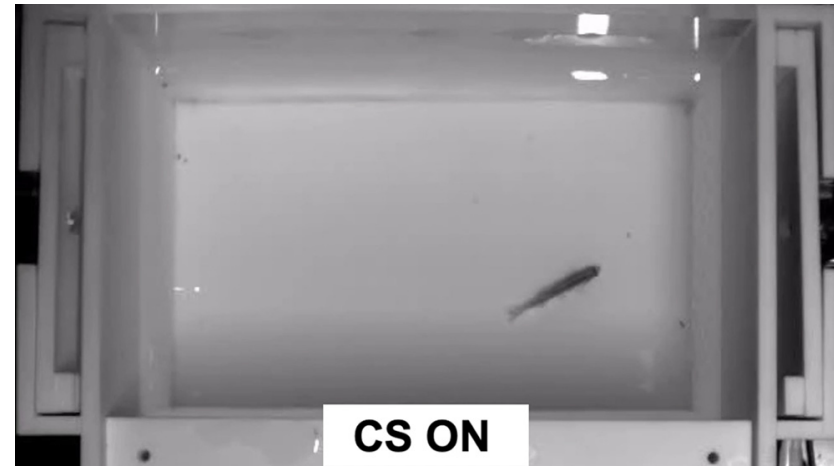

Movie 5. Pavlovian fear conditioning of an aldoca:BoTx fish. Behavior of an aldoca:BoTx non-learner fish in trial 1 of the test session is shown. The timing of the CS is indicated. Note that the fish did not move after the presentation of CS. [View online]

(Fig. 5). Although PC-ablated fish showed some abnormal swimming behaviors, they could respond to US in the same manner as wild-type fish (Figs. 2, 5). Similar to BoTx-expressing fish, PC-ablated fish showed reduced active avoidance conditioning. In contrast, NTR-MTZmediated ablation might induce an inflammatory response that exacerbated the cerebellar function, but it did not induce abnormal swimming, such as ataxia or rolling movements. Therefore, the BoTx-mediated inhibition of GCs/PCs and NTR-MTZ-mediated PC ablation were both able to induce defects in cerebellar neural circuits.

\section{The cerebellum is involved in active avoidance conditioning in zebrafish}

Early stage zebrafish larvae acquired operant conditioning in which they learned to turn their tail in the correct direction to obtain relief from an aversive heat stimulus (Lin et al., 2020). In that operant conditioning, cerebellar lesions affected the decision of which direction they would turn their tail (Lin et al., 2020). It is not clear whether the cerebellum is involved in other types of operant conditioning. In this study, we employed two-way active avoidance conditioning with adult zebrafish and genetically inhibited cerebellar neurons. Although the conditioning paradigm and fish ages are different, our findings clearly indicate that the zebrafish cerebellum is involved in active avoidance conditioning.

152B::BoTx fish expressed BoTx mainly in GCs of the medial CCe but only rarely in GCs in the LCa (Fig. 1C). This is consistent with previous data in which Gal4FF in gSA2AzGFF152B fish drove transgene expression mainly in GCs of the medial $\mathrm{CCe}$ in larvae and adult fish (Takeuchi et al., 2015; Matsuda et al., 2017). Since active avoidance conditioning, but not free swimming, was strongly suppressed in 152B::BoTx fish (Fig. 3), GCs in CCe play an important role in active avoidance conditioning but not in free swimming. Previously, it was reported that the inhibition of GC transmission in 152B::BoTx latestage larvae prolonged fear-conditioned bradycardia responses in which a set of neurons, most likely GCs, in the CCe became activated by the CS and were associated with conditioned bradycardia responses (Matsuda et al., 2017). The same or similar types of neurons might be 
involved in active avoidance conditioning. $\mathrm{As}^{2+}$ and voltage imaging of neurons in freely moving adult zebrafish are still difficult, we were unable to identify conditioning-associated neurons in active avoidance conditioning. Future studies with optical fibers or body-mounted fluorescence sensors that allow for the detection of immediate early gene expression, or virtual-reality conditioning assays, may reveal GCs and PCs that are associated with active avoidance conditioning.

The cerebellar vermis in mammals was reported to be involved in classical fear conditioning, such as autonomic bradycardia and freezing responses (Supple and Leaton, 1990; Supple and Kapp, 1993; Supple et al., 1993; Sacchetti et al., 2002). A previous study (Matsuda et al., 2017), as well as the current study, revealed that GCs in the CCe of the zebrafish cerebellum are involved in conditioned fear responses, including bradycardia and active avoidance. These findings suggest that the $\mathrm{CCe}$ of the zebrafish cerebellum has a function similar to that of the mammalian cerebellar vermis.

Cerebellar efferent neurons, which are eurydendroid cells, receive inputs directly from GCs and possibly integrate information of GCs (Harmon et al., 2020). Since BoTx-mediated inhibition of PC transmission or PC ablation in the adult cerebellum perturbed active avoidance conditioning (Figs. 4, 5), the transmission from PCs to eurydendroid cells is required for this conditioning. In goldfish, the activity of major PCs was suppressed whereas that of minor PCs was activated during classical fear conditioning (Yoshida and Kondo, 2012). Long-term depression (LTD) and long-term potentiation (LTP) in GC-PC synapses are involved in cerebellar learning (Ito and Kano, 1982; Salin et al., 1996; Lev-Ram et al., 2002; Sacchetti et al., 2004), although the contribution of LTD or LTP may depend on the type of learning. The inhibition or ablation of PCs might induce an imbalance of PC outputs, resulting in defective active avoidance behaviors. Future analysis of PC activity during conditioning will clarify this issue.

The habenula-raphe circuit and the medial zone of the dorsal telencephalon (Dm), which are thought to correspond to the amygdala in mammals, were involved in active avoidance conditioning in zebrafish (Aoki et al., 2013; Amo et al., 2014; Lal et al., 2018). In mammals, the amygdala plays essential roles in both classical fear conditioning and active avoidance conditioning (Duvarci and Pare, 2014; Herry and Johansen, 2014). Although a functional connection between the habenula and cerebellum was suggested (Lin et al., 2020), future studies on neural circuit connections between the cerebellum and the habenula/Dm are needed and will reveal what kind of information is transmitted to, and integrated in, the cerebellum.

\section{Roles of the cerebellum in Pavlovian fear and active avoidance conditioning}

Previously, BoTx-mediated inhibition of GCs did not perturb but rather prolonged CS-evoked bradycardia responses in zebrafish larvae, suggesting that cerebellar neural circuits control recovery from the fear-conditioned response (Matsuda et al., 2017). In this study, the inhibition of GCs or PCs suppressed CS-evoked Pavlovian panic responses, indicating a positive role of cerebellar neural circuits in the conditioned fear response. Why do the results of cerebellum inhibition differ between the two different classical conditioning paradigms? Although both are forms of classical fear conditioning in which CS-induced passive responses occur after conditioning, the bradycardia response is an autonomic response while the swimming response is a motor response. One possibility is that the two different conditioned responses are controlled by different neural circuits in the cerebellum although they share the same or similar sensory or integration systems, including the habenula nuclei and Dm. A different subpopulation of cerebellar neurons may control autonomic and motor responses. Total ablation of the $\mathrm{CCe}$ or anesthetic inhibition of the cerebellum impaired the acquisition of the conditioned bradycardia response in goldfish (Yoshida et al., 2004; Yoshida and Hirano, 2010), supporting the positive role of the entire cerebellum in classical fear conditioning. In 152B::BoTx fish, a specific GC subpopulation that functions in recovery from the conditioned fear response might be suppressed. Alternatively, the same cerebellar neurons may control both autonomic and motor responses in different manners: they regulate the timing of recovery from the freeze response while controlling the decision of the motor response. The elucidation of neurons whose activity was associated with each type of conditioned responses and/ or specific inhibition of GC or PC subpopulations in each conditioning paradigm will clarify neural circuits that control the conditioned autonomic and motor responses.

Both active avoidance and Pavlovian fear conditioning involve associative learning of the CS and US, and behavioral decisions based on them. We found that both of them were suppressed by PC or GC inhibition. Pavlovian fear conditioning was also established during active avoidance conditioning (Fig. 6). Although we could not determine the relationship between Pavlovian and active conditioning, it is possible that zebrafish first establish Pavlovian fear conditioning then, simultaneously or subsequently, move directionally to avoid US. In this scenario, the cerebellar neural circuits may control the initial process and possibly the decision-making of active avoidance conditioning. In summary, our findings indicate that the cerebellum plays active roles in active avoidance conditioning and provide a platform for understanding the mechanisms of conditioned fear responses.

\section{References}

Agetsuma M, Aizawa H, Aoki T, Nakayama R, Takahoko M, Goto M, Sassa T, Amo R, Shiraki T, Kawakami K, Hosoya T, Higashijima S, Okamoto $H$ (2010) The habenula is crucial for experience-dependent modification of fear responses in zebrafish. Nat Neurosci 13:1354-1356.

Ahrens MB, Li JM, Orger MB, Robson DN, Schier AF, Engert F, Portugues R (2012) Brain-wide neuronal dynamics during motor adaptation in zebrafish. Nature 485:471-477.

Aizenberg M, Schuman EM (2011) Cerebellar-dependent learning in larval zebrafish. J Neurosci 31:8708-8712. 
Akitake CM, Macurak M, Halpern ME, Goll MG (2011) Transgenerational analysis of transcriptional silencing in zebrafish. Dev Biol 352:191-201.

Amo R, Fredes F, Kinoshita M, Aoki R, Aizawa H, Agetsuma M, Aoki T, Shiraki T, Kakinuma H, Matsuda M, Yamazaki M, Takahoko M, Tsuboi T, Higashijima S, Miyasaka N, Koide T, Yabuki $\mathrm{Y}$, Yoshihara Y, Fukai T, Okamoto H (2014) The habenulo-raphe serotonergic circuit encodes an aversive expectation value essential for adaptive active avoidance of danger. Neuron 84:1034-1048.

Aoki T, Kinoshita M, Aoki R, Agetsuma M, Aizawa H, Yamazaki M, Takahoko M, Amo R, Arata A, Higashijima S, Tsuboi T, Okamoto H (2013) Imaging of neural ensemble for the retrieval of a learned behavioral program. Neuron 78:881-894.

Asakawa K, Suster ML, Mizusawa K, Nagayoshi S, Kotani T, Urasaki A, Kishimoto Y, Hibi M, Kawakami K (2008) Genetic dissection of neural circuits by Tol2 transposon-mediated Gal4 gene and enhancer trapping in zebrafish. Proc Natl Acad Sci USA 105:12551260.

Bae YK, Kani S, Shimizu T, Tanabe K, Nojima H, Kimura Y, Higashijima S, Hibi M (2009) Anatomy of zebrafish cerebellum and screen for mutations affecting its development. Dev Biol 330:406426.

Chang W, Pedroni A, Hohendorf V, Giacomello S, Hibi M, Köster RW, Ampatzis K (2020) Functionally distinct Purkinje cell types show temporal precision in encoding locomotion. Proc Natl Acad Sci USA 117:17330-17337.

Dohaku R, Yamaguchi M, Yamamoto N, Shimizu T, Osakada F, Hibi M (2019) Tracing of afferent connections in the zebrafish cerebellum using recombinant rabies virus. Front Neural Circuits 13:30.

Duvarci S, Pare D (2014) Amygdala microcircuits controlling learned fear. Neuron 82:966-980.

Harmon TC, McLean DL, Raman IM (2020) Integration of swimmingrelated synaptic excitation and inhibition by olig2(+) eurydendroid neurons in larval zebrafish cerebellum. J Neurosci 40:3063-3074.

Hashimoto M, Hibi M (2012) Development and evolution of cerebellar neural circuits. Dev Growth Differ 54:373-389.

Herry C, Johansen JP (2014) Encoding of fear learning and memory in distributed neuronal circuits. Nat Neurosci 17:1644-1654.

Hibi M, Shimizu T (2012) Development of the cerebellum and cerebellar neural circuits. Dev Neurobiol 72:282-301.

Hibi M, Matsuda K, Takeuchi M, Shimizu T, Murakami Y (2017) Evolutionary mechanisms that generate morphology and neuralcircuit diversity of the cerebellum. Dev Growth Differ 59:228-243.

Ito $M(2005)$ Bases and implications of learning in the cerebellumadaptive control and internal model mechanism. Prog Brain Res 148:95-109.

Ito M (2006) Cerebellar circuitry as a neuronal machine. Prog Neurobiol 78:272-303.

Ito M (2008) Control of mental activities by internal models in the cerebellum. Nat Rev Neurosci 9:304-313.

Ito M, Kano M (1982) Long-lasting depression of parallel fiberPurkinje cell transmission induced by conjunctive stimulation of parallel fibers and climbing fibers in the cerebellar cortex. Neurosci Lett 33:253-258.

Kani S, Bae YK, Shimizu T, Tanabe K, Satou C, Parsons MJ, Scott E, Higashijima S, Hibi M (2010) Proneural gene-linked neurogenesis in zebrafish cerebellum. Dev Biol 343:1-17.

Kotajima H, Sakai K, Hashikawa T, Yanagihara D (2014) Effects of inferior olive lesion on fear-conditioned bradycardia. Neuroreport 25:556-561.

Lal P, Tanabe H, Suster ML, Ailani D, Kotani Y, Muto A, Itoh M, Iwasaki M, Wada H, Yaksi E, Kawakami K (2018) Identification of a neuronal population in the telencephalon essential for fear conditioning in zebrafish. BMC Biol 16:45.
Lev-Ram V, Wong ST, Storm DR, Tsien RY (2002) A new form of cerebellar long-term potentiation is postsynaptic and depends on nitric oxide but not cAMP. Proc Natl Acad Sci USA 99:8389-8393.

Lin Q, Manley J, Helmreich M, Schlumm F, Li JM, Robson DN, Engert F, Schier A, Nöbauer T, Vaziri A (2020) Cerebellar neurodynamics predict decision timing and outcome on the single-trial level. Cell 180:536-551.e17.

Matsuda K, Yoshida M, Kawakami K, Hibi M, Shimizu T (2017) Granule cells control recovery from classical conditioned fear responses in the zebrafish cerebellum. Sci Rep 7:11865.

Nojima H, Rothhämel S, Shimizu T, Kim CH, Yonemura S, Marlow FL, Hibi M (2010) Syntabulin, a motor protein linker, controls dorsal determination. Development 137:923-933.

Pisharath H, Rhee JM, Swanson MA, Leach SD, Parsons MJ (2007) Targeted ablation of beta cells in the embryonic zebrafish pancreas using E. coli nitroreductase. Mech Dev 124:218-229.

Sacchetti B, Baldi E, Lorenzini CA, Bucherelli C (2002) Cerebellar role in fear-conditioning consolidation. Proc Natl Acad Sci USA 99:8406-8411.

Sacchetti B, Scelfo B, Tempia F, Strata P (2004) Long-term synaptic changes induced in the cerebellar cortex by fear conditioning. Neuron 42:973-982.

Salin PA, Malenka RC, Nicoll RA (1996) Cyclic AMP mediates a presynaptic form of LTP at cerebellar parallel fiber synapses. Neuron 16:797-803.

Skinner BF (1984) The evolution of behavior. J Exp Anal Behav 41:217-221.

Sternberg JR, Severi KE, Fidelin K, Gomez J, Ihara H, Alcheikh Y, Hubbard JM, Kawakami K, Suster M, Wyart C (2016) Optimization of a neurotoxin to investigate the contribution of excitatory interneurons to speed modulation in vivo. Curr Biol 26:2319-2328.

Supple WF Jr, Leaton RN (1990) Cerebellar vermis: essential for classically conditioned bradycardia in the rat. Brain Res 509:17-23.

Supple WF Jr, Kapp BS (1993) The anterior cerebellar vermis: essential involvement in classically conditioned bradycardia in the rabbit. J Neurosci 13:3705-3711.

Supple WF Jr, Sebastiani L, Kapp BS (1993) Purkinje cell responses in the anterior cerebellar vermis during Pavlovian fear conditioning in the rabbit. Neuroreport 4:975-978.

Tabor KM, Bergeron SA, Horstick EJ, Jordan DC, Aho V, PorkkaHeiskanen T, Haspel G, Burgess HA (2014) Direct activation of the Mauthner cell by electric field pulses drives ultrarapid escape responses. J Neurophysiol 112:834-844.

Takeuchi M, Matsuda K, Yamaguchi S, Asakawa K, Miyasaka N, Lal P, Yoshihara Y, Koga A, Kawakami K, Shimizu T, Hibi M (2015) Establishment of Gal4 transgenic zebrafish lines for analysis of development of cerebellar neural circuitry. Dev Biol 397:1-17.

Tanabe K, Kani S, Shimizu T, Bae YK, Abe T, Hibi M (2010) Atypical protein kinase $C$ regulates primary dendrite specification of cerebellar Purkinje cells by localizing Golgi apparatus. J Neurosci 30:16983-16992.

Valente A, Huang KH, Portugues R, Engert F (2012) Ontogeny of classical and operant learning behaviors in zebrafish. Learn Mem 19:170-177.

Yoshida M, Hirano R (2010) Effects of local anesthesia of the cerebellum on classical fear conditioning in goldfish. Behav Brain Funct 6:20.

Yoshida M, Kondo H (2012) Fear conditioning-related changes in cerebellar Purkinje cell activities in goldfish. Behav Brain Funct 8:52.

Yoshida M, Okamura I, Uematsu K (2004) Involvement of the cerebellum in classical fear conditioning in goldfish. Behav Brain Res 153:143-148. 